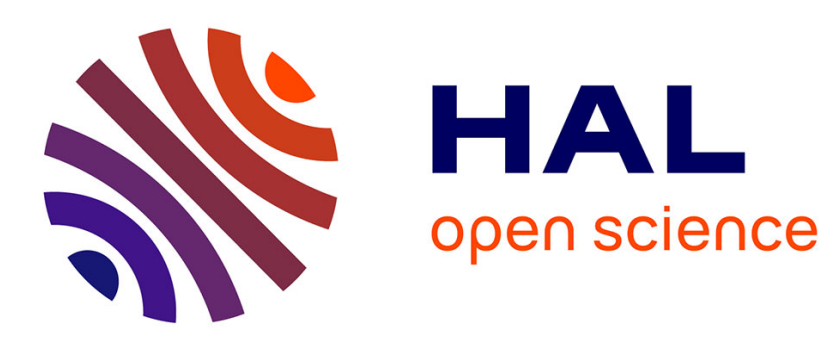

\title{
L'ébauche d'un nouvel enseignement de la langue écrite à la veille des réformes éducatives de 1872
}

Christian Galan

\section{To cite this version:}

Christian Galan. L'ébauche d'un nouvel enseignement de la langue écrite à la veille des réformes éducatives de 1872. Ebisu - Études Japonaises , 1999, 22 (1), pp.77-124. 10.3406/ebisu.1999.1027. hal-02399514

\section{HAL Id: hal-02399514 https://hal.science/hal-02399514}

Submitted on 9 Dec 2019

HAL is a multi-disciplinary open access archive for the deposit and dissemination of scientific research documents, whether they are published or not. The documents may come from teaching and research institutions in France or abroad, or from public or private research centers.
L'archive ouverte pluridisciplinaire HAL, est destinée au dépôt et à la diffusion de documents scientifiques de niveau recherche, publiés ou non, émanant des établissements d'enseignement et de recherche français ou étrangers, des laboratoires publics ou privés. 


\title{
L'ébauche d'un nouvel enseignement de la langue écrite à la veille des réformes éducatives de 1872
}

\section{Christian Galan}

\section{Résumé}

C'est à partir d'une critique de la conception de l'enseignement de la langue (lecture et écriture) et du savoir qui réduisait l'étude à l'acte de lire, conception dominante durant toute l'époque d'Edo, que, à partir des années 1860, les tenants des études occidentales remettent en cause l'éducation classique dans son ensemble. Un nouveau type de manuels de langue clairement destinés à des débutants voit alors le jour, et cela avant même l'établissement d'un système éducatif national. Rédigés à partir de nouveaux principes pédagogiques et d'une réflexion-critique sur la langue écrite japonaise, ces manuels vont fonder l'enseignement moderne de la lecture et de l'écriture. Dans cet article, on s'attachera plus particulièrement à ceux de Fukuzawa Yukichi, Yanagawa Harukage et Furukawa Masao, qui sont parmi les plus représentatifs de ces manuels charnières entre l'éducation classique et l'éducation moderne.

\begin{abstract}
From the 1860s onwards, the supporters of Western studies called into question traditional education as a whole. They were critical of the concept upon which the teaching of the language (reading and writing) had been based as well as the preeminence given to reading as the only means of access to knowledge. Thus, even before the founding of a national education system, a new type of textbook clearly aimed at beginners appeared for the teaching of the language. Based on some novel pedagogical principles and critical considerations of the written Japanese language, these books established the modern teaching of reading and writing. In this article, we will consider those of Fukuzawa Yukichi, Yanagawa Harukage and Furukawa Masao, as their textbooks are some of the most representative works marking the transition from classical to modern education.
\end{abstract}

\section{Citer ce document / Cite this document :}

Galan Christian. L'ébauche d'un nouvel enseignement de la langue écrite à la veille des réformes éducatives de 1872. In: Ebisu, n²2, 1999. pp. 77-124.

doi : 10.3406/ebisu.1999.1027

http://www.persee.fr/doc/ebisu_1340-3656_1999_num_22_1_1027

Document généré le 16/10/2015 


\title{
L'EBAUCHE D'UN NOUVEL ENSEIGNEMENT DE LA LANGUE ECRITE À LA VEILLE DES RÉFORMES ÉDUCATIVES DE $1872^{1}$
}

\author{
Christian GALAN
}

\begin{abstract}
Résumé
C'est à partir d'une critique de la conception de l'enseignement de la langue (lecture et écriture) et du savoir qui réduisait l'étude à l'acte de lire, conception dominante durant toute l'époque d'Edo, que, à partir des années 1860 , les tenants des études occidentales remettent en cause l'éducation classique dans son ensemble. Un nouveau type de manuels de langue clairement destinés à des débutants voit alors le jour, et cela avant même l'établissement d'un système éducatif national. Rédigés à partir de nouveaux principes pédagogiques et d'une réflexion-critique sur la langue écrite japonaise, ces manuels vont fonder l'enseignement moderne de la lecture et de l'écriture. Dans cet article, on s'attachera plus particulièrement à ceux de Fukuzawa Yukichi, Yanagawa Harukage et Furukawa Masao, qui sont parmi les plus représentatifs de ces manuels charnières entre l'éducation classique et l'éducation moderne.
\end{abstract}

${ }^{1}$ Cet article vient à la suite de celui publié dans le $n^{\circ} 18 \mathrm{~d}^{\prime} E b i s u$ et intitulé "L'enseignement de la lecture à la veille de la promulgation du Gakusei (1872) - La méthode classique », automne-hiver 1998 (b), p. 5-47.

EBISU 22, Automne-hiver 1999, Maison Franco-Japonaise, Tôkyô, p. 77-124. 


\title{
Outlines for a New Teaching of the Written Language on the Eve of the Educational Reforms of 1872
}

From the 1860s onwards, the supporters of Western studies called into question traditional education as a whole. They were critical of the concept upon which the teaching of the language (reading and writing) had been based as well as the preeminence given to reading as the only means of access to knowledge. Thus, even before the founding of a national education system, a new type of textbook clearly aimed at beginners appeared for the teaching of the language. Based on some novel pedagogical principles and critical considerations of the written Japanese language, these books established the modern teaching of reading and writing. In this article, we will consider those of Fukuzawa Yukichi, Yanagawa Harukage and Furukawa Masao, as their textbooks are some of the most representative works marking the transition from classical to modern education.

学制公布前夜の読み方の教育一吾き言葉の新しい教育への草案

\author{
クリスチャン・ガラン \\ トゥールーズ第 2 大学助教授
}

1860 年代から、西欧流学習の信奉者たちが古典的な教育の全体を問題視した のは、（曹き言葉、話し言葉を含めた）言語教育の構想と江戸期を通じて支配的で あった学習を読み取りという行為に還元してしまう知というものの批判をもとにし てであった。はっきりと初心者を対象とした新しい言語の教科曹はこのようにして 世に出たのであり、これは国としての教育制度の確立以前のことでさえあった。日 本語の書き言葉に対する批判的な考察からの新しい教育方針をもとにして茟かれた これらの教科書が、読み方と青き方の近代的な教育を確立していくことになるので ある。本論文では、とりわけ福沢諭吉、柳河春䔖、古川正雄の教科曺をとりあげた。 これらは古典的な教育と近代教育の境目に位置する教科書を代表しているからであ る。 


\section{Introduction}

Les quatre-vingts premières années du XIX ${ }^{e}$ siècle, qui sont aussi celles où les principes de mérite et de réussite l'emportent progressivement sur la hiérarchie et l'origine sociale, apparaissent comme l'une des périodes les plus riches et les plus libres de la réflexion et de la pratique éducatives au Japon. La pensée éducative dominante est empreinte d'une double philosophie : confucéenne, héritée des écoles des fiefs, et utilitariste, héritée des terakoya 寺子屋². Pédagogiquement, l'essentiel des pratiques repose sur la mémorisation, qui est quasi unanimement considérée comme le meilleur des apprentissages.

Les premières remises en cause de la conception classique de l'éducation, avant même l'introduction des idées occidentales - même si celle-ci en hâtera l'ampleur - sont le fait de jeunes intellectuels qui éprouvent à son encontre une réaction quasi viscérale de rejet et lui reprochent, à partir des années 1850 , son inadéquation à la réalité du monde contemporain ${ }^{3}$. Le " courant de la pensée éducative utilitariste", jitsuri shugi no kyôiku shisô 実利主義の教育思想, se développe à la fin du shôgunat et au début de l'ère Meiji (1868-1912) à l'initiative de ceux qui ont fait le voyage en Occident. Pour la plupart en rupture avec les études classiques dès avant leur départ, ces jeunes occidentalistes ont vu leurs convictions s'affirmer au cours de leur(s) voyage(s) et prônent à leur retour une éducation de l'individu, libérale, pratique, rationnelle et respectueuse de l'esprit scientifique. La nouvelle

${ }^{2}$ Cf. Christian Galan, "Le paysage scolaire à la veille de la Restauration de Meiji - écoles et manuels ", Ebisu - études japonaises, $n^{\circ} 17$, printemps-été 1998 (a), p. 5-48.

${ }^{3}$ Une première remise en cause des pratiques traditionnelles avait été le fait des confucianistes opposés au néo-confucianisme qui, tels Itô Jinsai 伊藤 仁盾 (1627-1705) ou Ogyû Sorai 获生徂来 (1666-1728), revendiquèrent un retour à la pensée originelle du confucianisme et doublèrent leur critique philosophique d'une remise en cause des méthodes d'enseignement. Leur critique n'alla toutefois pas jusqu'à remettre en cause les principes fondamentaux de la conception classique du savoir et leurs propositions pour une autre pédagogie concernaient essentiellement les étudiants les plus avancés. 
éducation qu'ils réclament est à leurs yeux la condition sine qua non pour que le Japon devienne une nation libre constituée d'hommes libres.

Fukuzawa Yukichi 福沢諭吉 (1835-1901), le plus fameux d'entre eux, préconise une éducation fondée sur les "études pratiques", jitsugaku 実学, terme par lequel il désigne à la fois les savoirs et savoirfaire ayant une utilité directe dans la vie quotidienne et les sciences - en fait tout ce qui ne relève pas du domaine des études classiques, chinoises ou japonaises. La philosophie du Gakusei 学制 de 1872 , le texte fondateur du système éducatif moderne ${ }^{4}$, relèvera de ce courant de pensée des " études pratiques ", renforcé par l'utilitarianism anglo-saxon qui considérait l'instruction comme devant préparer à la vie de tous les jours et permettre à chacun de s'élever dans la société. Ce dernier point de vue fut d'autant plus facilement accepté par les Japonais qu'il ne leur était pas étranger puisque, même s'il n'avait pas été exactement théorisé en ces termes, il était la raison même d'exister des terakoya dans lesquelles les parents envoyaient leurs enfants quérir un savoir pratique, utile pour leur vie future d'adulte. Ceux qui, à l'instar de Fukuzawa, prônaient l'utilitarisme à la veille de la Restauration le faisaient simplement, à présent, en visant le devenir du pays tout entier et non plus celui des individus.

La " continuité dans la tradition " qui caractérise le début de Meiji en matière de méthodes d'enseignement ne doit cependant pas cacher le fait que, d'un point de vue historique strictement

${ }^{4}$ Promulgué le 4 août 1872, le Gakusei restera en vigueur jusqu'en 1879. Le système éducatif qu'il met en place comporte trois niveaux : élémentaire, secondaire et universitaire. Tous les établissements scolaires passent sous la responsabilité unique du département de l'Education qui fixe les programmes et supervise les enseignements et les enseignants. Considérée comme la clef de voûte de l'ensemble, l'éducation élémentaire devient l'objet de toutes les attentions. Les principes de base du Gakusei apparaissent clairement dans son préambule : rupture avec le système précédent d'essence confucéenne, indépendance et prospérité du pays clairement associées à l'élévation du niveau d'éducation de la population, nécessité d'une éducation véritablement nationale, priorité accordée au développement et à la formation de l'individu, obligation de mettre en place une éducation "pratique " non coupée des réalités de la vie quotidienne et favorisant la diffusion des sciences. 
chronologique, une nouvelle réflexion sur les contenus d'enseignement et plus particulièrement sur ceux de l'enseignement de la lecture et de l'écriture s'est fait jour dès avant 1872 . Elle s'inscrivait à la fois dans le cadre de la remise en cause fondamentale des principes d'éducation classiques, mais également, et de façon tout aussi profonde, dans celui d'une réflexion plus large sur la langue écrite. Cette nouvelle réflexion, qui n'a pas entraîné de changements notables sur le plan des pratiques pédagogiques avant la promulgation du Gakusei, est cependant à l'origine de la publication, dans les années 1865-1870, de manuels de langue d'un nouveau genre qui marquent une rupture avec les ouvrages scolaires de l'époque d'Edo (1603-1868) et annoncent les futurs manuels pour débutants des années 1872-1879. Présenter ces manuels dans la seconde partie d'un article consacré aux pratiques pédagogiques de l'époque d'Edo permet de souligner, notamment au travers du cas de Fukuzawa Yukichi, l'importance du débat sur la langue et l'influence que celui-ci eut sur l'enseignement de la lecture et de l'écriture, tout en insistant sur le caractère non-officiel de leur élaboration, c'est-à-dire avant même que n'apparaisse tout embryon d'une organisation nationale de l'éducation. La première étape vers l'enseignement moderne de la langue est le fait d'individus certes préoccupés par les questions d'éducation, mais qui ne sont pas à proprement parler des pédagogues ou de "purs " spécialistes de l'enseignement de la langue à l'école élémentaire. L'acquisition de la lecture et de l'écriture par tous est avant tout, pour eux, un critère de modernisation et de civilisation et fait partie, à ce titre, de leurs nombreuses préoccupations - parfois fort éloignées des problèmes d'éducation ou de langue. Bien que peu utilisés dans les classes où, dans l'immense majorité des cas, les maîtres continuaient à avoir recours aux anciens matériaux de l'époque d'Edo, les manuels qu'ils rédigent sont extrêmement importants au regard de la réflexion pédagogique et de l'évolution des conceptions de l'enseignement de la langue.

${ }^{5}$ Cf. Christian Galan, "Les manuels de langue au lendemain de la Restauration de Meiji - Les innovations de la période du décret sur l'éducation ", Cipango, n 8, automne 1999, p. 215-257. 
Les manuels de Fukuzawa Yukichi : une réflexion sur la langue doublée d'un souci pédagogique

Parmi les nombreux écrits de Fukuzawa Yukichi consacrés à l'éducation figurent plusieurs manuels scolaires ou ouvrages ayant été utilisés comme tels. Citons le Shôchû bankoku ichiran 掌中万国一覧 (Petit atlas du monde, 1869), le Dômô oshie gusa 童蒙教草 (Manuel d'éthique pour les jeunes, 1872), le Kyûri zukai 窎理図解 (Livre de physique illustré, 1868), le Sekai kuni zukushi 世界国尽 (Tous les pays du monde, 1869), qui a été utilisé en tant que manuel de lecture et d'écriture, ainsi que beaucoup d'autres dont le Keimô tenarai no fumi 啓蒙手習之文 (Introduction à l'écriture, 1871), et le Moji no oshie 文字之 教 (Leçons d'écriture, 1873). Nous allons nous intéresser tout particulièrement à ces deux derniers qui concernent directement l'enseignement de la lecture et de l'écriture et furent utilisés en tant que manuels scolaires de niveau élémentaire.

\section{Le Keimô tenarai no fumi (1871)}

Le Keimô tenarai no fumi se présente sous la forme de deux fascicules publiés en mai 1871 et réédités en juin $1873^{6}$. L'ensemble était précédé d'une préface rédigée en mars 1871, dont on trouvera la traduction ci-après.

Voici ce qui est écrit dans le livre Description générale des écoles en Occident [ 学校軌镇 Gakkô kihan] traduit par mon collègue Obata [Obata Jinzaburô 小幡甚三郎 (1845-1873)] : " nous voulons, en ouvrant largement la voie de l'éducation, non pas faire de tous nos compatriotes des politiciens ou des érudits, mais les mener vers le bien, et ce faisant renforcer le gouvernement et rendre prospère la nation. Si l'on suit cet objectif, il faut que l'enseignement dans nos

${ }^{6}$ En tant que manuel d'écriture, cet ouvrage a été calligraphié en "grandes lettres " par un élève de Fukuzawa particulièrement doué pour la calligraphie, Uchida Shinsai 内田晋在 (1848-?). Source : Fukuzawa Yukichi zenshû 福沢諭吉全 集 (Euvres complètes de Fukuzawa Yukichi), vol. 3, Tôkyô, Iwanami shoten 岩波搂店, 1959, p. 1-20. La traduction qui suit se trouve p. 3-4. 


\section{L'ÉBAUCHE D'UN NOUVEL ENSEIGNEMENT DE LA LANGUE ECRITE}

écoles touche la multitude en visant vers le bas plutôt que d'être élevé mais réservé à une élite.

Parmi les pays occidentaux, la Russie est le pays où l'éducation est la moins répandue : il n'y a qu'un étudiant pour 77 habitants. Dans un pays comme la Hollande en revanche où l'éducation est très répandue, il y a un étudiant pour 8 habitants. Il faut aujourd'hui que le Japon instaure une loi sur l'éducation et, en essayant de se situer entre la Russie et la Hollande, prenne pour objectif le chiffre d'environ 1 étudiant pour 42 habitants. La population du Japon se montant actuellement à 40 millions d'habitants, 1/42 de ce nombre représente 940000 étudiants. Pour fonder rapidement de grandes écoles afin d'éduquer ces élèves et offrir un enseignement de qualité à ces 940000 étudiants, les dépenses seront considérables. Même si les frais de nourriture et de vêtements sont pris en charge par les parents, les salaires des professeurs et du personnel, l'entretien des bâtiments et les frais de livres et d'équipement matériel représentent, si l'on calcule le coût moyen par étudiant, la somme de 100 ryô par étudiant et par an, non compris la nourriture et les vêtements. 100 ryô multipliés par 940 000, cela fait 94 millions de ryô. Tel est le coût de l'éducation pour le pays.

Le pays est il assez prospère pour cela ? Tel est, en mon for intérieur, l'objet de mes craintes. Aussi, en suivant une politique d'avancées progressives, il faut ouvrir la voie de l'éducation extrêmement modestement et commencer par les maîtres d'écriture [tenarai shishô 手習師匠]. On pourra ainsi faire faire de grandes économies au pays tout en diffusant largement l'instruction. Cependant, l'enseignement donné par les maîtres d'écriture s'est fait jusqu'ici en ne tenant pas compte de l'impérieuse nécessité des études pratiques [jitsugaku] et en se limitant simplement aux lettres de félicitations pour les diverses cérémonies, mariages, festivals et autres, aux lettres de condoléances, à la littérature ou encore à la poésie classique chinoise ou japonaise. Ce qui aujourd'hui, dans un monde qui s'ouvre à la civilisation, est une perte de temps.

Aussi, après discussion avec mes collègues, j'ai fabriqué et publié un livre de modèles [tehon 手本] pour l'écriture en langue vulgaire 
qui débute par le tableau des 47 signes de l'iroha ${ }^{7}$ et les noms des provinces, à la suite desquels $j$ 'ai introduit différentes idées prises dans des ouvrages occidentaux. Mon væu est que les enfants de cinq à six ans, en comprenant le sens des textes tout en étudiant les signes, puissent accéder plus facilement à leurs futures études. Ce fascicule devrait aider les enseignants d'écriture et être utile à l'éducation en général. Nous en serions très heureux.

\section{Mai $1871 \quad$ Fukuzawa Yukichi}

Ce texte montre bien comment deux sujets apparemment sans relation directe, la gestion administrative du système éducatif et la pédagogie de l'écriture, pouvaient être liés pour un intellectuel des années 1860-70. Le raisonnement était le suivant : pour le bien du pays, tout le monde doit être éduqué ; or, ne serait-ce que pour atteindre un "niveau international moyen ", l'investissement financier est considérable; le Japon n'en ayant actuellement pas les moyens, il faut progresser petit à petit et la première étape doit être le recrutement d'un nombre suffisant de "maîtres d'écriture "; cependant, comme ceux-ci ont la fâcheuse habitude de n'enseigner que des choses sans rapport avec la vie quotidienne, moi, Fukuzawa Yukichi, j'ai rédigé à leur intention ce manuel qui débute de façon traditionnelle et se poursuit en présentant quelques connaissances d'ordre général, utiles, empruntées à des ouvrages occidentaux.

La priorité de Fukuzawa, ce sont les " maîtres d'écriture ", c'està-dire les enseignants des terakoya dont la tâche consistait essentiellement à l'origine à " enseigner les signes " ${ }^{8}$. Fukuzawa les appelle d'ailleurs de leur ancien nom, tenarai shishô. Ils doivent être en nombre suffisant pour permettre au peuple de parvenir au premier stade de l'étude : la maîtrise de l'écrit. Fukuzawa reste ainsi fidèle à

${ }^{7}$ L'iroha ou iroha uta ( syllabes (comme notre " abc ") est le plus ancien des syllabaires. De caractère mnémotechnique, il se présente sous la forme d'un poème d'inspiration bouddhique de la fin du $X^{e}$ siècle où chaque syllabe n'apparaît qu'une seule fois.

$$
{ }^{8} \text { Cf. C. Galan (1998-b), op. cit., p. 32-40. }
$$




\section{L'ÉBAUCHE D'UN NOUVEL ENSEIGNEMENT DE LA LANGUE ÉCRITE}

la conception classique qui faisait de l'écriture le premier des enseignements. Il perpétue de même l'usage consistant à utiliser cet enseignement comme véhicule de l'instruction morale et de connaissances diverses : il le "réactualise " simplement au profit des connaissances occidentales et d'un discours moral plus adapté à sa conception du citoyen moderne'.

Les idées que Fukuzawa expose dans ce texte sont au centre du débat sur l'éducation des années 1860-70. Lui-même les reprendra l'année suivante dans son "Appel à l'étude ", Gakumon no susume 学 問のす ১ : le but de l'éducation n'est pas seulement de faire des savants mais des hommes de bien, dont l'existence profitera à la nation ; l'école doit être ouverte au plus grand nombre ; elle ne doit pas être élitiste ni réservée à quelques-uns. Ces idées étaient déjà en filigrane dans le “Serment en cinq articles 》, Gokajô no goseimon 五䇢条の御誓文, prononcé le 6 avril 1868 par l'empereur Meiji (1852-1912) et qui énonçait les intentions du nouveau pouvoir en matière d'éducation.

Ce qui est particulièrement intéressant dans cette préface, c'est que l'éducation et, si l'on suit bien le raisonnement de Fukuzawa, l'enseignement de la lecture et de l'écriture, y sont considérés comme importants et prioritaires au nom du bien et de la prospérité de la nation.

Cependant, chaque homme doit posséder les qualités et les capacités appropriées à sa position dans la société. Or pour acquérir de telles qualités, il est nécessaire de connaître le principe des choses, il

${ }^{9}$ La pratique généralisée de l'époque d'Edo consistant à utiliser les cours d'écriture pour enseigner " autre chose " se perpétua bien après la promulgation du Gakusei, et cela malgré l'établissement d'une matière tenarai censée concerner exclusivement cet enseignement. Cette double vocation des cours d'écriture qui apparaît très clairement dans l'ouvrage de Fukuzawa est également perceptible dans le Tenarai kôyô bunshô 習字公用文章 (Leçons d'écriture avec des documents officiels, 1873) de Kuroda Yukimoto 黑田行元 ou encore dans le Shôgaku tenarai nôgyô ôrai 小学習字農業往来 (Órai des travaux agricoles - écriture pour l'école élémentaire, 1879) d'Itô Keishû 伊藤桂州, publiés vers la même époque et qui lient très fortement enseignement de l'écriture et connaissances nécessaires à la vie quotidienne. Sur le terme d'Ôrai, nous renvoyons infra, note 35. 
est nécessaire d'avoir appris à lire. L'éducation est ainsi devenue un besoin urgent. [...]

Il n'y a pas dans la société de personnes plus misérables et plus méprisables que les ignorants et les illettrés. L'ignorance extrême conduit à la disparition de tout sentiment de honte. Ceux qui, à cause de leur ignorance, sombrent dans la pauvreté et sont tenaillés par le froid, la faim, oublient qu'ils en sont là par leur propre faute et s'en prennent fort injustement aux gens riches qui les entourent. En dernière extrémité, ils vont jusqu'à former des factions et à user de violence dans des protestations de masse ou des insurrections. Doit-on dire qu'ils ont perdu toute honte ou qu'ils se moquent des lois?

\section{(L'Appel à l'étude, Livre premier) $^{10}$}

Le sens profond que revêtira par la suite l'enseignement officiel de la lecture, c'est-à-dire considéré dans le cadre du futur système éducatif national, transparaît dans ces lignes : il est bon pour la nation que chaque individu soit instruit et sache lire. La réflexion sur cet enseignement est ainsi, dès le début, menée dans une perspective d'intérêt national et non pas individuel. C'est ce message que le Gakusei reprendra et amplifiera et cette conception de «l'alphabétisation »du pays aura des effets durables - perceptibles encore de nos jours - sur les pratiques pédagogiques liées à l'enseignement de la lecture.

Délaissant les problèmes financiers, Fukuzawa poursuit avec ce qui représente à ses yeux le principal obstacle à la mise en place $d$ 'une instruction véritablement nationale : la coupable négligence dont font preuve depuis toujours les " maîtres d'écriture " à l'égard de ce qu'il nomme les "études pratiques". C'est que ceux-ci se contentent d'enseigner l'écriture aux enfants au travers de textes dénués de tout intérêt immédiat. On retrouve là le Fukuzawa "pro-yôgaku " (洋学, les études occidentales), ou plutôt "anti-kangaku » (漢学, les

${ }^{10}$ Fukuzawa Yukichi, Gakumon no susume 学問のす 、め (L'Appel à l'étude), 初編，Tôkyô, Iwanami bunko 岩波文庫, 1991, p. 16-17. Nous avons proposé une traduction intégrale de ce texte dans l'ouvrage collectif Cent Ans de pensée au Japon (sous la direction $\mathrm{d}^{\prime} Y$ ves-Marie Allioux), Arles, éditions Philippe Picquier, 1996, vol. 2, p. 7-20. 


\section{L'ÉBAUCHE D'UN NOUVEL ENSEIGNEMENT DE LA LANGUE ECRITE}

études chinoises), qui, dans cette période de sa vie, s'en prend chaque fois qu'il le peut aux études classiques et confucéennes. Précisant sa pensée, il écrit :

Par «étude ", je n'entends pas ici les seules études littéraires qui consistent à connaître des caractères chinois complexes, lire des textes anciens difficiles à interpréter ou encore composer des poèmes japonais et écrire des vers chinois, études qui n'ont aucun intérêt pratique dans notre société. Il s'agit certes là d'activités agréables à l'esprit humain et non dénuées de valeur en elles-mêmes, mais elles ne sont sans doute pas aussi essentielles que l'affirment les confucianistes et les spécialistes des études nationales depuis des siècles. [...]

Il faut [...] apprendre les quarante-sept signes du syllabaire japonais, assimiler la façon de rédiger une lettre, de tenir un livre de compte, savoir utiliser un abaque ou une balance. Au fur et à mesure que l'on progresse, le nombre des matières augmente. Il y a la géographie, guide des climats et des régions $d u$ Japon et de tous les pays du monde; la physique, science qui s'intéresse à ce qui constitue l'univers et étudie la fonction de chacun des éléments qui le composent ; l'histoire, qui établit la chronologie détaillée des différentes époques et examine les événements passés et présents des pays du monde; l'économie, qui explique la gestion des moyens d'existence de l'individu, de la famille et de la nation tout entière; la morale, enfin, exposant les grands principes naturels qui doivent régir les actes de chaque individu, ainsi que ses relations avec les autres et son comportement au sein de la société.

Pour étudier chacun de ces domaines, il est nécessaire de lire des traductions de livres occidentaux. Dans la plupart des cas, connaître les syllabaires japonais se révèle suffisant. Pourtant si l'on a affaire à un élève qui présente de réelles dispositions pour les lettres, il faut le rendre capable de lire aussi les originaux en langue occidentale.

(L'Appel à l'étude, Livre premier) ${ }^{11}$

${ }^{11}$ Fukuzawa Y., op. cit., p. 12. 


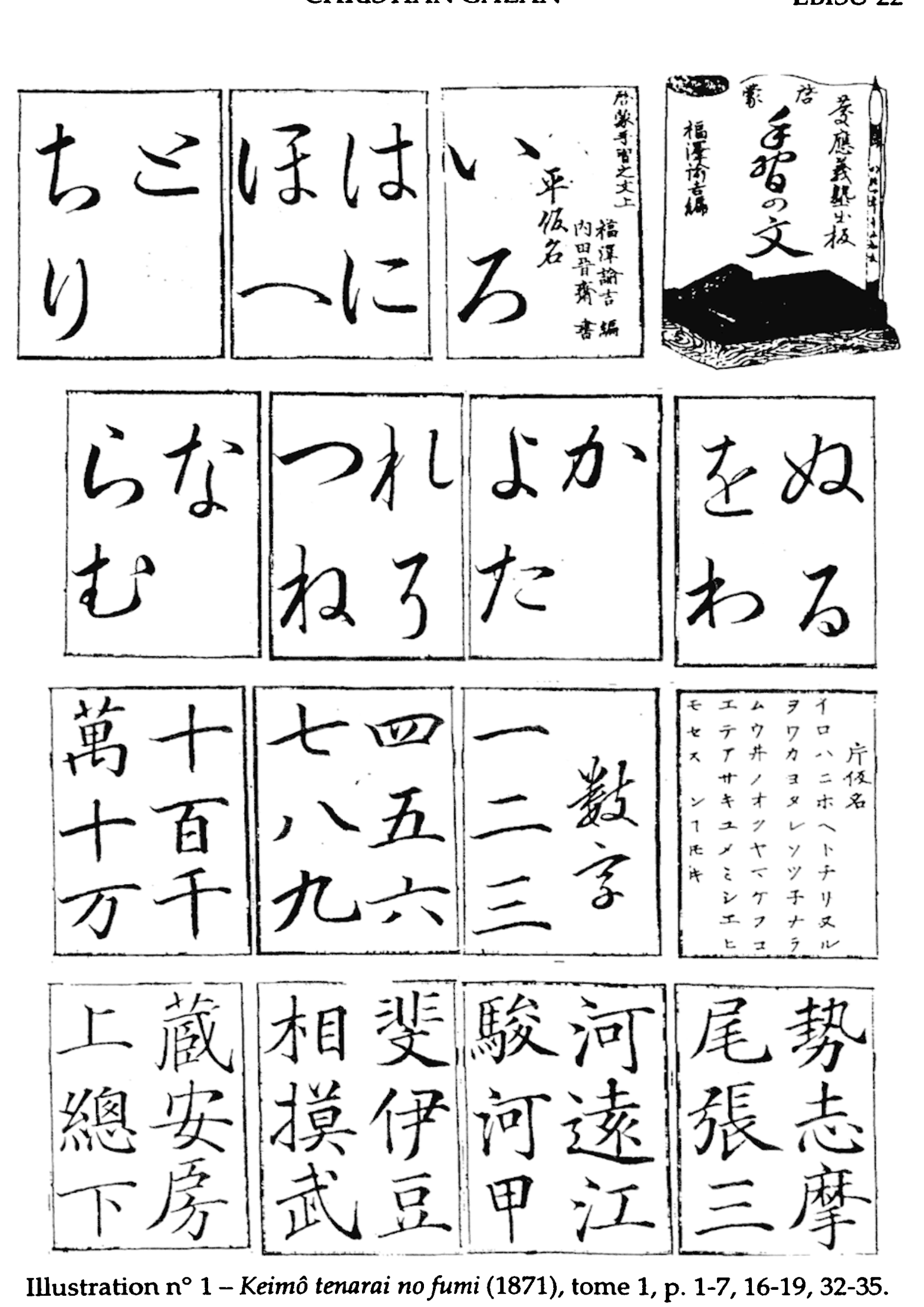


C'est ce même Fukuzawa qui revendique, dans la préface de Keimô tenarai no fumi, "Introduction à l'écriture ", d'écrire dans un style simple et "vulgaire". Ce qui ne l'empêche pas de s'exprimer en "gestionnaire ", cours d'économie de l'éducation à l'appui, sinon en penseur de la modernité, dans un manuel scolaire destiné à des enfants de 5 à 7 ans. C'est là une caractéristique qu'il partage avec les autres " nouveaux manuels" de l'époque qui, plus que de simples ouvrages scolaires, contenaient dans leur préface des développements théoriques ou philosophiques, souvent militants, s'adressant aux adultes qu'ils cherchaient à convaincre, bien plus qu'aux enfants à qui le manuel était destiné.

Venons-en maintenant au contenu du manuel lui-même. Le premier fascicule (107 pages) se compose de 7 leçons (voir illustration $\left.n^{\circ} 1\right)^{12}$ :

1 - l'iroha en hiragana ( 15 pages de 2 à 4 signes chacune)

2 - l'iroha en katakana (1 page)

3 - les chiffres en kanji ( 5 pages)

4 - les dix " tiges célestes " du calendrier chinois ( 2 pages et demie)

5 - les 12 signes horaires ou « branches terrestres » de ce même calendrier ( 2 pages et demie)

6 - les régions du Japon ( 40 pages, 6 kanji maximum par page tracés en style carré)

7 - l'univers : espace et temps ( 35 pages, 6 kanji maximum par page tracés en style semi-cursif)

Et le second fascicule ( 49 pages) de 5 leçons, entièrement en style semi-cursif :

1 - géographie mondiale (20 pages, phrases écrites en caractères de taille moyenne)

2 - dialogue sur les sciences ( 32 pages, phrases écrites en caractères de petite taille)

3 - lettre pour demander conseil au sujet des études occidentales (2 pages, caractères de très petite taille)

4 - réponse à cette lettre (4 pages)

${ }^{12}$ La préface couvrait quatre pages et était suivie d'une table des matières de deux pages. 
5 - définitions des "matières " occidentales: lecture, géographie, mathématiques, physique, histoire, économie et éthique (11 pages en style carré).

Les cinq premières leçons ${ }^{13} \mathrm{du}$ premier fascicule se présentent sous la forme d'une énumération de kana qui occupe les deux premières, puis d'un répertoire de kanji réparti entre les trois suivantes. La sixième leçon est composée de la liste des noms des régions du Japon, la plupart des kanji étant particulièrement complexes. Quant à la dernière leçon, ainsi que toutes celles du second fascicule, il s'agit de textes de vulgarisation (phrases/expressions), le dernier consistant en un inventaire des " matières scolaires occidentales " avec pour chacune d'entre elles une présentation sommaire (domaines couverts et contenus) et les points les plus importants les concernant. Qu'il s'agisse du contenu ou de la progression, Fukuzawa s'aligne ainsi sur les terakoya : iroha (hiragana > katakana), chiffres, mots, expressions, etc.

Fukuzawa ouvre sa présentation des matières occidentales par la «lecture». Il utilise à son endroit le terme de tokuhon 讀本, qui signifie littéralement "livre de lecture » et qui est la traduction du mot américain reader. Ce genre de manuels, qui n'existait pas auparavant au Japon, fut importé d'Occident ${ }^{14}$ dans les années 1867-68.

${ }^{13}$ Nous avons employé ici le terme de "leçon " mais celui de " chapitre " conviendrait peut-être mieux étant donné que, si nous ne savons pas à quel rythme ni comment le contenu était abordé - ou devait l'être dans l'esprit de Fukuzawa -, il est peu probable qu'une de ces "leçons " ait tenu en seul cours. Nous avons cependant retenu le terme de leçon par analogie avec les autres ouvrages de l'époque servant de manuels.

${ }^{14}$ Fukuzawa en avait lui-même rapporté quelques-uns de ses divers voyages en Occident et sa bibliothèque, déposée au Centre d'études Fukuzawa de l'université de Keiô, Keiô gijuku Fukuzawa kenkyû sentâ 慶隹義熟福沢研究七 ンター, en conserve encore certains : E. H. Butler, The New American Primary Speller, Philadelphia, E. H. Butler \& Co.'s new American series, 1872, [cote FY37] ; Epes Sargent (1813-1880) [\& Amasa May], The New American Fourth Reader, Philadelphia, E. H. Butler \& Co.'s new American series, 1871, [cote FY42(4)] ; Epes Sargent (1813-1880) [\& Amasa May], The New American Fifth Reader, Philadelphia, E. H. Butler \& Co.'s new American series, 1871, [cote FY42(5)] ; Harry's Little Lessons, Harry's Reading Book, Philadelphia, Porter \& Coates, (18??), 2 tomes en 1, [cote FY19]. 


\section{L'ÉBAUCHE D’UN NOUVEL ENSEIGNEMENT DE LA LANGUE ÉCRITE}

Fukuzawa explique ainsi :

C'est un livre qui, avec des phrases très faciles, présente les premières notions des différents domaines d'étude. A côté de l'étude de ce manuel de lecture, on doit étudier aussi le livre de grammaire. Si on ne connait pas la grammaire, on ne peut ni lire un livre ni comprendre ce qui est écrit et on ne parvient pas à communiquer par l'intermédiaire de la langue. Les gens incapables de communiquer par la langue ne different en rien des muets. ${ }^{15}$

Il est clair qu'avec le Keimô tenarai no fumi, Fukuzawa ne parvient pas vraiment à rompre avec les pratiques anciennes et que son manuel s'inscrit dans la droite ligne des "modèles d'écriture ", tehon, utilisés jusque-là dans les terakoya. Il reprend l'idée de la pédagogie classique qui veut que ce soit par la pratique de la copie au pinceau que les enfants, en répétant encore et encore le tracé des mêmes modèles, apprennent "par la main » à la fois le tracé des signes, la lecture, la technique de la composition écrite et les connaissances contenues dans les textes. Le contenu " pédagogique " du manuel est de fait bien moins novateur que sa préface. Il est même beaucoup moins original que d'autres manuels parus à la même époque et sur lesquels nous reviendrons plus loin. Si ces derniers partagent avec celui de Fukuzawa certains défauts (la présentation "à vide » ou hors contexte d'un grand nombre de mots ou de signes, sans souci de leur difficulté ou de leur fréquence d'apparition dans les textes ; la primauté accordée à l'écrit sur l'oral ; la lecture considérée, au travers de l'écriture, comme une simple accumulation d'éléments graphiques, etc.), ils réussissent en effet beaucoup mieux dans leur volonté de rompre (tant sur le fond que sur la forme) avec l'enseignement traditionnel. Enfin, on peut encore reprocher à Fukuzawa de ne pas tenir compte, dans les textes qu'il propose, des capacités réelles des enfants auxquels il s'adresse ni de leurs préoccupations propres. La langue, simple et " populaire » comme le souhaitait son auteur, n'étant tout de même guère adaptée à des enfants aussi jeunes.

${ }^{15}$ Deuxième fascicule, dernière leçon. Fukuzawa Yukichi zensh $\hat{u}$, vol. 3, op. cit., p. 18. 
Ce manuel reflète, en fait, très bien la situation de la réflexion sur l'enseignement au Japon dans les années 1860-1870 : la volonté de rompre avec les conceptions anciennes de l'enseignement est réelle et clairement affichée, mais sur le plan pédagogique, la modélisation ne suit pas : un modèle et/ou des solutions de remplacement tardent à être trouvés et mis en place. L'enfant n'est pas encore pris en compte en tant que tel. Malgré sa préface, le Keimô tenarai no fumi de Fukuzawa appartient, pédagogiquement, davantage à l'époque d'Edo qu'à la nouvelle ère Meiji.

\section{Le Moji no oshie (1873)}

Entre la parution du Keimô tenarai no fumi (mai 1871) et celle du Moji no oshie (juin 1873), lui aussi consacré à la lecture/écriture, divers événements majeurs se sont produits qui ont profondément marqué l'histoire de l'éducation du Japon : un département de l'Education (nouveau Monbushô 文部省) a été créé en septembre 1871 et un an plus tard, en septembre 1872, le Gakusei, a été promulgué, dotant le Japon de son premier système éducatif national et moderne. Bien que le nouveau manuel de Fukuzawa soit ultérieur, il nous a toutefois semblé important de le présenter ici afin de mieux cerner l'évolution de la pensée de son auteur et, plus généralement, celle de la réflexion sur la langue et son influence sur les nouveaux manuels.

Les trois fascicules du Moji no oshie 文字之教 ${ }^{16}$, littéralement "L'enseignement des signes ", furent publiés une première fois en juin 1873, puis réédités en 1876 et vraisemblablement aussi en 1878 ou 1880. Le premier fascicule (48 pages), Daiichi moji no oshie 第一文字 之教, se composait de 40 leçons toutes bâties sur le même modèle : une liste de 4 à 8 mots écrits en gros caractères en style carré et transcrits chacun à l'aide soit d'un ou de deux kanji (plus rarement de trois), soit d'un kanji (ou de deux) suivi(s) d'okurigana, kana marquant un suffixe fonctionnel verbal, adjectival ou encore adverbial, le tout précédant de courtes phrases ou expressions écrites en petits caractères et dans lesquelles ces mots étaient réutilisés (voir illustration $n^{\circ} 2$ ).

${ }^{16}$ Fukuzawa Yukichi zenshû, vol. 3, op. cit., p. 553-611. 
L'ÉBAUCHE D'UN NOUVEL ENSEIGNEMENT DE LA LANGUE ÉCRITE
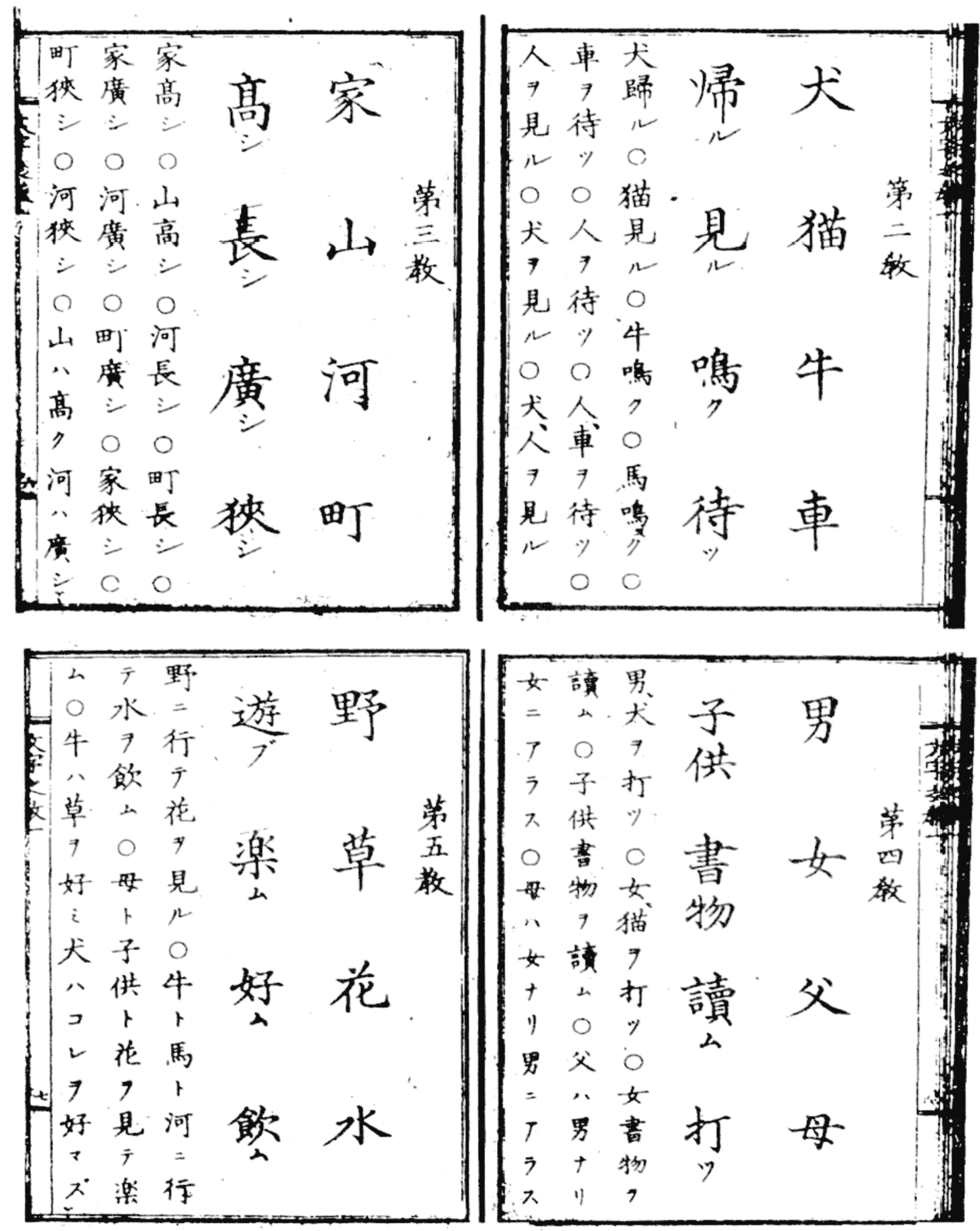

Illustration $n^{\circ} 2-$ Moji no oshie (1873), tome 1, leçons 2 à 5 . 
Le deuxième fascicule (60 pages), Daini moji no oshie 第二文字之 教, se composait de 33 leçons bâties exactement sur le même modèle que celles du premier fascicule, mais légèrement étoffées : une dizaine de nouveaux mots par leçon, chacun transcrit à l'aide de 1 à 3 kanji tracés en style carré, et des textes de réemploi un peu plus longs.

Quant au troisième fascicule (60 pages), Moji no oshie - furoku 文 字之教付録 (appendice), il se voulait, comme l'indiquait son sous-titre, Tegami no fumi 手文, une introduction en 27 leçons (toujours bâties sur le même modèle, mais cette fois tracées en style semi-cursif) à $l^{\prime}$ " écriture de la correspondance" (vocabulaire, conventions, style). Une de ces leçons se composait d'un texte "de style et de contenu imbéciles " destiné à montrer aux élèves ce qu'il fallait absolument éviter de faire. Le procédé était audacieux : montrer ce qu'il ne faut pas faire plutôt que ce qu'il faut faire, et c'était sans doute une des toutes premières fois qu'il était utilisé dans un manuel de ce genre.

Voyons à présent la préface générale du manuel, figurant au début du premier fascicule ${ }^{17}$ :

Bien que l'utilisation conjointe des kana et des kanji qui a cours au Japon soit extrêmement peu pratique, celle des caractères chinois dans les écrits étant très ancienne et répandue dans tout le pays, il serait tout aussi peu pratique, à présent, de supprimer ces derniers du jour au lendemain. Comme il semble, face à ces deux inconvénients, que les kanji bien que peu pratiques soient toutefois utiles, la solution de les abandonner complètement, tout en étant souhaitable, apparaît aujourd'hui très difficile à réaliser.

Attendre que la situation évolue ne signifie pas attendre à ne rien faire et on doit dès maintenant créer progressivement les conditions de l'abandon des kanji. Pour cela, il faut, lorsqu'on écrit, éviter le plus possible d'utiliser des caractères difficiles. Une fois ceux-ci éliminés, les deux à trois mille restant sont bien suffisants. Dans ces trois

${ }^{17}$ Une courte préface au début du tome trois reprend l'explication de la méthode, mais cette fois adaptée à la correspondance. En résumé : si l'apprenant a bien étudié les fascicules 1 et 2 du Moji no oshie et s'il maîtrise leur contenu, il doit à présent s'exercer à rédiger des lettres. Le principe des leçons est le même que dans les deux fascicules précédents. 


\section{L'ÉBAUCHE D'UN NOUVEL ENSEIGNEMENT DE LA LANGUE ÉCRITE}

fascicules, le nombre des mots transcrits en caractères chinois est inférieur à mille, sans que cela ne cause une quelconque gêne. Mêler ainsi kanji et kana ne posera pas non plus de problèmes aux érudits. Il suffit simplement de veiller à ne pas utiliser de caractères difficiles, comme le faisaient les confucianistes par le passé. De plus, c'est un jeu parfaitement inutile que de faire souffrir les enfants en leur faisant lire [en sodoku 素読 ${ }^{18}$ ] des classiques chinois afin de les entraîner à aimer les textes difficiles.

Si pour des mots tels que ishiya [医者, médecin, transcrit à l'époque en kana : いしや] ou ishiya [石屋 / いしや, tailleur de pierre] les kanji sont plus pratiques que les kana, distinguer par des kanji différents 上る，登る，昇る et 攀る [qui se lisent tous noboru, et traduisent tous l'idée de monter, de lever] n'est pas du tout pratique. Que le singe monte [等る] dans l'arbre ou que l'homme gravisse [登 Ђ] une montagne, comme dans tous les cas cela se dit noboru en japonais, il est plus pratique d'utiliser les kana que les kanji. Pour les mots variables, l'usage des kana est, de loin, préférable.

Bien que lire [en sodoku, déchiffrer donc] ne soit absolument pas difficile lorsqu'il s'agit de distinguer entre eux des kanji faciles, il vaut mieux, plutôt que de déchiffrer les caractères, s'efforcer de comprendre le sens du texte. Aussi le présent ouvrage a-t-il été rédigé dans le but de faire parvenir les enfants à la compréhension $d u$ sens des textes lus. Voyons à présent comment enseigner cela.

Dans cet ouvrage, on appellera "titre" ce qui figure en gros caractères et "texte" ce qui est imprimé en petits caractères. Les "titres " sont composés des " mots-graines " avec lesquels seront fabriqués les "textes". On enseignera d'abord aux enfants la lecture en sodoku du "titre", puis on en enseignera le sens; on laissera ensuite l'enfant déchiffrer librement le texte en petits caractères et en

${ }^{18}$ La lecture appelée sodoku 素読 (ou su yomi), « lecture-déchiffrage » ou " lecture à haute voix ", littéralement " lecture simple ", constituait la première étape de l'enseignement de la lecture dans la méthode classique. La recherche de la compréhension était complètement absente de ce type de lecture qui reposait sur l'idée qu'en répétant encore et encore, on finissait par parvenir " naturellement " au sens, selon l'adage chinois qui dit que : « si on lit cent fois un texte le sens surgit de lui-même ", dokusho hyappen $i$ onozukara tsûzu 読書百遍意自ら通ず。 
comprendre le sens à son idée. A l'école, le professeur enlèvera les livres aux enfants assis à leur place et distribuera feuilles et pinceaux, puis lui seul regardant le livre, il copiera au tableau les seuls kanji $d u$ " titre ". Il expliquera aux enfants le sens des signes, puis lira le texte écrit en petits caractères et leur fera écrire les sons qu'il vient de lire. Pour la leçon 4, par exemple, il écrira au tableau les signes du "titre": 男 homme，女 femme，父 père，母 mère, etc., puis il interrogera sur leur sens. Ensuite lorsqu'il lira le texte écrit en petits caractères : "L'homme frappe le chien", les enfants, s'ils regardent le tableau en écoutant les sons verront les signes transcrivant les mots "homme" et "frapper ", mais pas " chien". Les enfants qui ont une bonne mémoire se souviendront du signe transcrivant "chien " vu dans la deuxième leçon et l'écriront, les enfants qui ne l'auront pas mémorisé ne sauront pas écrire ce kanji L'enseignant pourra ainsi évaluer le travail des enfants et leur donner une note.

Quand on augmente le nombre de pages d'un livre, son prix augmente d'autant et il devient difficile à utiliser dans les écoles élémentaires. Aussi, si l'on a réduit ici le nombre de pages en réduisant les textes écrits en petits caractères, ce n'est pas pour épargner des tracas à l'auteur, c'est pour faire faire des économies à ceux qui achèteront le livre. Toutefois, même si les textes écrits en petits caractères sont courts, comme il y a déjà dans l'ouvrage des éléments ordonnés dans les " titres", le professeur peut improviser instantanément des textes. S'il pense par exemple que la phrase de la leçon deux "L'homme voit le chien " est insuffisante, il peut sur le champ en inventer d'autres, telles que "Il regarde le chien et le chat", "La vache regarde la voiture, mais la voiture ne regarde pas la vache", etc. Il peut fabriquer ainsi des phrases dans sa tête, les dire à haute voix et les faire copier aux élèves, selon la méthode indiquée précédemment. Il ne doit cependant utiliser, le deuxième jour par exemple, que les signes des " titres" des leçons 1 et 2. De même le troisième jour, il ne doit absolument pas utiliser d'autres signes que ceux vus jusqu'à la troisième leçon. S'il ne peut toutefois faire autrement que d'utiliser des signes non-étudiés, il devra les écrire aussitôt au tableau.

Quand on avance progressivement en suivant la méthode cidessus, [les élèves] ne sont pas rebutés comme avec les textes difficiles 


\section{L'ÉBAUCHE D'UN NOUVEL ENSEIGNEMENT DE LA LANGUE ÉCRITE}

des classiques chinois et en renonçant à la lecture de type sodoku des Quatre Classiques et des Cinq Livres, on aborde la lecture et la rédaction autrement. C'est là mon voeu le plus cher.

\section{Août $1873, l^{\prime}$ Auteur ${ }^{19}$}

L'intérêt principal de ce texte réside dans le fait qu'après avoir présenté les inconvénients et les avantages respectifs des kana et des kanji et réclamé une limitation de ces derniers, Fukuzawa propose une rupture fondamentale avec l'enseignement de la lecture pratiqué jusque-là : l'abandon pur et simple de la lecture de type sodoku, pratique qui selon lui torture inutilement les enfants et ne va pas à l'essentiel, le sens. L'idée est si novatrice qu'elle mettra plusieurs décennies à s'imposer vraiment.

Si le plaidoyer pour l'abandon des kanji est fréquent chez les intellectuels de l'époque qui tentent, dans des textes plus ou moins enflammés, de convaincre leurs contemporains de cette nécessité ${ }^{20}$, la démarche de Fukuzawa et ses propositions pratiques sont tout à fait originales. En apparence beaucoup moins radical que certains (Maejima Hisoka 前島密 (1835-1919) ${ }^{21}$ par exemple), Fukuzawa va beaucoup plus loin sur le plan de la cohérence et du concret de ses propositions, qui sont par ailleurs immédiatement applicables : écrire dans un style simple $^{22}$, auto-limitation des kanji, enseignement d'une lecture centrée

${ }^{19}$ Fukuzaiva Yukichi zenshû, vol. 3, op. cit., p. 555-557.

${ }^{20} C^{\prime}$ est le discours que tiennent notamment les membres du groupe Meirokusha 明六社 (Le groupe de l'an six de Meiji) auquel appartient Fukuzawa. Premier groupe d'intellectuels de l'après-Meiji, ses membres, tels Nishi Amane 西周 (1829-1897) ou Mori Arinori 森有礼 (1847-1889), se consacrèrent à la diffusion des idéaux de bunmei kaika 文明開化, les "Lumières" japonaises. Sur le débat sur la langue et sur le système d'écriture, on se reportera aux ouvrages ou articles de Pascal Griolet : La Modernisation du Japon et la réforme de son écriture, Paris, POF, 1985 ; Les Japonais face à leur écriture, politiques et polémiques de 1900 à nos jours, Paris, thèse de doctorat INALCO, 1990 ; ainsi que celui de Nanette Twine, Language and the Modern State, London and New York, Routledge, 1991.

${ }^{21}$ Cf. P. Griolet (1985), op. cit., p. 16-22.

${ }^{22}$ Le style "vulgaire " dont parle Fukuzawa est davantage un style 
sur le sens et non plus simple déchiffrage, etc. L'évolution ultérieure du système d'écriture du japonais et de l'enseignement de la lecture donnera raison à son réalisme et fera de lui un précurseur.

«Faire souffrir » les enfants en leur faisant lire en sodoku des textes aussi difficiles que les classiques chinois est pour Fukuzawa un exercice parfaitement inutile. Plus que l'abandon de l'outil «kanji», c'est son utilisation qu'il veut réformer. Pour lui, le monopole exercé sur l'éducation par l'ensemble " confucianistes-sodoku-kanji» doit disparaître parce qu'il entraîne les apprenants vers un savoir de "luxe" - ou de " pacotille" - et les tient écartés des vraies connaissances et des réalités de leur époque. Fukuzawa s'élève avant tout contre la conception classique du savoir qui réduit toute étude à la seule lecture de livres, telle que la définit par exemple Emura Hokkai 江邨北海(1713-1788) ${ }^{23}$. Il s'en était clairement expliqué dans le deuxième livre de Gakumon no susume, quelques mois auparavant, en novembre $1873^{24}$ :

[...] Pour élargir le champ de ses connaissances et de ses expériences, il faut soit écouter ce que disent les autres, soit chercher à comprendre par soi-même, soit encore lire un certain nombre de livres. Si la maîtrise de la lecture est indispensable à l'étude, c'est cependant une grosse erreur que de croire, comme on le fait depuis les temps les plus anciens, qu'étudier se limite au seul acte de lire. Les signes de l'écrit ne sont que les outils de l'étude. Ils sont comparables aux marteaux et aux scies qu'on utilise pour construire une maison. Ces instruments sont indispensables, mais connaître uniquement leur nom sans savoir comment on bâtit n'a jamais autorisé quiconque à se dire charpentier. Pour la même raison, ceux qui savent lire mais sont incapables de discerner l'esprit des choses ne méritent pas le nom de savants. Tous

littéraire simplifié qu'un style véritablement "familier». Sur le style de Fukuzawa on se reportera avec intérêt à l'ouvrage de N. Twine, op. cit., p. 53-56, 82-85, 111-115, 224-227.

${ }^{23}$ Cf. C. Galan (1998-b), op. cit., p. 30.

${ }^{24}$ Fukuzawa Yukichi, op. cit., p. 19-20. Nous avons proposé une traduction intégrale de ce texte dans la revue Daruma - Revue internationale d'études japonaises, $n^{\circ} 2$, automne 1997, p. 259-266. 


\section{L'ÉBAUCHE D'UN NOUVEL ENSEIGNEMENT DE LA LANGUE ECRITE}

ceux dont on dit qu'ils ont lu Confucius sans jamais rien y comprendre sont dans ce cas.

Un homme pourra réciter par cour un de nos classiques japonais comme le $\mathrm{Kojiki}^{25}$, s'il ne connaît pas le cours actuel du riz, on dira qu'il est ignare en économie. Un autre peut avoir percé le sens profond des classiques chinois, s'il n'entend rien aux lois du commerce et se révèle incapable d'accomplir correctement une transaction, on dira qu'il est un incompétent en matière de gestion. Un autre enfin, au bout de dures années de labeur et après y avoir laissé beaucoup d'argent, aura beau être devenu un spécialiste éminent des études occidentales, s'il n'arrive pas à assurer sa propre subsistance, ce n'est qu'un sot qui vit coupé des sciences vivantes de son temps. De tels individus ne doivent pas être pris pour autre chose que des grossistes de l'écrit. Leurs capacités, leurs mérites, ne dépassent pas ceux d'un bon dictionnaire de cuisine et loin d'être de quelque utilité pour leur pays, ce sont plutôt des parasites qui entravent la bonne marche de son économie. L'économie domestique fait partie des études, la comptabilité fait partie des études et parvenir à une bonne compréhension de son époque fait aussi partie des études. Pourquoi alors réserver le mot étude à la seule lecture des livres, qu'ils soient japonais, chinois ou occidentaux?

J'ai intitulé mon ouvrage "L'appel à l'étude ", mais mon appel ne se limite absolument pas à la seule pratique de la lecture. [...]

\section{Comme le souligne très justement Pascal Griolet :}

Bien avant que la linguistique occidentale ne soit introduite au Japon et qu'Ueda Kazutoshi [上田万年 (1867-1937)] ne reprenne à son compte la conception selon laquelle l'écriture est secondaire par rapport à la langue, Fukuzawa compare déjà les signes de l'écriture à des instruments et refuse de leur reconnaître une valeur absolue.

${ }^{25}$ Le Kojiki 古事記 (Chroniques des choses anciennes) est un recueil de mythes, de légendes, de généalogies et d'événements historiques, compilé à partir de 711 pour tenir lieu d' " Histoire officielle ". C'est le premier grand classique japonais. 
Le problème de l'écriture est posé, comme chez Maejima, en terme d'éducation et de science. Mais là où Maejima prend pour cible les signes et le code lui-même, Fukuzawa s'attaque surtout à leurs utilisateurs et tous ceux qui se fourvoient dans des études stériles et perdent le contact réel, obnubilés par la fascination qu'exercent les signes écrits. Telle devait être l'image que se faisait une grande part de la population non savante de gakumon, "l'étude " ou "la recherche», et celle que donnaient d'eux-mêmes les lettrés ${ }^{26}$.

Enfin, dans le dix-septième livre de Gakumon no susume (novembre 1876) Fukuzawa réhabilite la " parole » qui dans la culture classique était considérée comme inférieure et secondaire face à l'écrit :

Il faut premièrement étudier la langue. L'écriture est un moyen de communiquer très puissant et il ne faut pas négliger le soin qui doit être apporté à la correspondance ou à la rédaction d'un texte, mais dans le contact direct entre individus, il n'y a rien de plus puissant que la parole pour transmettre sans détour le fond de sa pensée. C'est pourquoi il faut être capable de parler avec aisance et rapidité. [...] les Japonais doivent travailler à utiliser intelligemment leur langue et à faire des progrès dans l'art de s'exprimer".

Accordant ses actes à ses idées, Fukuzawa a rédigé un manuel dans lequel, pour la première fois, le nombre des kanji a été volontairement limité en tenant compte, pour la première fois également, de leur fréquence d'apparition à l'écrit ${ }^{28}$.

Considérons maintenant la méthode d'enseignement qu'il propose au travers de ce manuel. Tout d'abord un constat : Fukuzawa

${ }^{26}$ P. Griolet (1985), op. cit., p. 80.

${ }^{27}$ Traduction de P. Griolet, ibid., p. 83-84.

${ }^{28}$ Comme le fait encore remarquer P. Griolet (op. cit., 1985, p. 82) : « [...] parmi les 802 signes qu'il choisit d'enseigner en premier, 717 furent de nouveau retenus pour la liste standard qui fut élaborée en 1946. Ce manuel met ainsi en évidence qu'il existe une sorte de noyau de caractères chinois fondamentaux que tout le monde peut manier aisément, alors que d'autres font appel à un savoir spécialisé, à une forme d'érudition. " 
parle de lecture dans un livre destiné à l'apprentissage de l'écriture. Sur ce point au moins, il y a continuité avec son manuel précédent et avec la pratique de l'époque d'Edo qui organisaient l'apprentissage de la lecture à l'intérieur ou à partir de celui de l'écriture. Toutefois, même s'il s'agit d'abord d'un manuel d'écriture, les deux enseignements de la lecture et de l'écriture y apparaissent, sans que cela soit dit explicitement, comme liés et simultanés : c'est une nouveauté, ils étaient jusque-là considérés comme distincts et consécutifs.

Fukuzawa innove sur de nombreux autres points. Pédagogiquement, il propose : l'enseignement collectif (le maître s'adresse à la classe dans son ensemble et utilise le tableau noir), une progression stricte allant du facile au difficile et une utilisation souple du manuel (le maître propose d'autres phrases qui n'y figurent pas). L'influence occidentale est sensible et réelle: Fukuzawa a vu ce qui se passait dans les écoles occidentales qu'il a visitées au cours de ses voyages et il sait que l'Américain Marion McCarrel Scott (1843-1922) présente, depuis quelques mois lorsqu'il écrit ces lignes, la "méthode d'enseignement simultané ", issei kyôju hô 一斉教授法, dans laquelle l'enseignant ne s'adressait plus à un seul élève à la fois, mais à toute une classe.

Bien qu'il ne parle pas de l'enseignement des kana, la progression qu'il propose laisse supposer qu'il en fait un apprentissage préalable à l'étude de ses deux fascicules. Le contenu de ces derniers fait en effet apparaître une conception de l'apprentissage de la langue écrite reposant sur un apprentissage cumulatif progressant par synthèse du simple au complexe. Cet apprentissage débute par les kana et se poursuit non pas par les kanji pris un à un, comme c'était en général le cas jusque-là, mais par des mots dont le sens est aussitôt enseigné, puis par des phrases de réemploi où apparaissent kana et kanji mêlés. Sur ce point encore, Fukuzawa fait œuvre de précurseur et il faudra attendre plus de vingt-cinq ans pour que les Japonais abandonnent les méthodes syllabiques pures, qui vont dominer les deux premières décennies de l'ère Meiji, au profit des word methods (méthodes partant du mot et non plus de la syllabe) - encore que, répétons-le, on n'ait pas ici 
d'éléments qui nous informent sur sa conception de l'apprentissage des kana.

Tout en étant fondée sur l'intuition que l'enseignement de la lecture devait se centrer sur le sens, la conception que Fukuzawa a de cet enseignement présente dans ses autres aspects une couleur plus classique : il ne demande pas de partir du vécu des enfants, et c'est le maître, ou le manuel, qui fixe le rythme d'apprentissage ; il insiste sur le sens, mais les phrases qu'il propose et surtout leur "fabrication » synthétique par accumulation et synthèse d'éléments nouveaux (en petit nombre) parmi d'autres déjà vus, ne sont pas véritablement porteuses de sens... tel qu'on l'entend aujourd'hui en didactique de la lecture. Sur un autre plan, Fukuzawa - comme ses aînés - semble faire dépendre l'apprentissage de la lecture de la seule mémoire des signes, ce qui semble un peu en contradiction avec son souci du sens, le sens (la compréhension) devant aider à mémoriser et non le contraire. Les idées qu'il avance représentent cependant un progrès énorme par rapport à la période précédente où les signes, à ce niveau d'étude, étaient étudiés sans grand souci de leur signification.

L'analyse successive du Tenarai no fumi et du Moji no oshie met bien en lumière la rupture qui existe entre ces deux manuels : rupture épistémologique au niveau de la conception de la lecture, rupture au niveau didactique. Là où le premier ne proposait aucune innovation et se trouvait en décalage avec le discours novateur de sa préface, le second avance une méthode concrète et pratique qui s'appuie sur une conception nouvelle, originale pour l'époque, de l'acte de lire. Fukuzawa recentre par ailleurs le Moji no oshie sur la lecture et l'écriture proprement dites et n'en fait pas un manuel de vulgarisation véhiculant des connaissances sur d'autres matières. Cette conception d'un manuel de lecture centré uniquement sur la lecture mettra pourtant plus de cinquante ans à s'imposer et jusqu'à la fin des années 1930, lecture, histoire, géographie et sciences seront étroitement liées au sein des manuels de langue des six premières années de l'école élémentaire.

Par rapport au premier manuel enfin, apparaît dans le Moji no oshie un souci plus grand de l'apprenant qui se manifeste par des textes plus simples, mis davantage à la portée de jeunes enfants. Avec ce manuel, la réflexion commence à se déplacer de la langue elle-même 
(objet de l'apprentissage) vers l'enfant (sujet/acteur de ce même apprentissage).

Le manuel de 1873 repose indéniablement sur une réflexion concernant la lecture plus avancée que celle du manuel de 1871. A quoi est due cette amélioration ? En grande partie, probablement, à l'évolution de la réflexion de Fukuzawa lui-même, à son intuition aussi et à son réalisme. Egalement sans doute, en ce qui concerne l'étalement de l'apprentissage du simple au complexe ou la prise en compte de l'enfant, aux idées occidentales introduites durant cette période. Notons cependant que les méthodes de lecture occidentales de l'époque (essentiellement syllabiques et qui vont avoir une influence déterminante sur les méthodes de lecture japonaises) n'accordaient pas vraiment d'importance au sens et mettaient surtout l'accent sur le déchiffrage et l'oralisation. Ce qui tendrait à prouver que sur ce point au moins la réflexion de Fukuzawa s'est développée hors de toute influence extérieure.

La " méthode Fukuzawa ", tout en présentant encore un grand nombre de caractéristiques qui, du point de vue de l'histoire générale de l'enseignement de la lecture, l'apparentent aux " anciennes" méthodes, apparaît au Japon comme tout à fait novatrice au niveau de la pratique scolaire bien sûr, mais surtout au niveau des préoccupations de son auteur : la compréhension, le sens, le souci de donner une finalité pratique à la lecture/écriture (illustré par le troisième fascicule où l'on apprend à rédiger des lettres).

L'étude de ces deux manuels, et des autres écrits de Fukuzawa sur la langue, permet de saisir, à partir d'un cas très concret, l'évolution de la réflexion sur la lecture qui a lieu durant les quelques années où se met en place une nouvelle conception de l'éducation. La pensée utilitariste, à la base du développement des terakoya durant l'époque $\mathrm{d}^{\prime} E d o$, est étendue à l'ensemble de l'éducation : derrière ces deux manuels, il y a l'objectif de savoir rédiger des lettres nécessaires à la vie sociale et professionnelle moderne. Des terakoya subsiste également l'idée qu'entre la lecture et l'écriture, c'est l'enseignement de l'écriture qui est premier, mais dans le même temps la maîtrise de la langue orale apparaît comme un préalable à celle de la langue écrite. L'enfant commence à être " réellement " pris en compte. En ce qui concerne 
l'enseignement proprement dit, la pratique de la lecture de type sodoku est remise en cause et une nouvelle définition de la lecture s'ébauche : lire, c'est parvenir au sens. La volonté d'enseigner au travers d'une méthode progressive, procédant du simple au complexe apparaît. Le soin que prend Fukuzawa pour expliquer la démarche à suivre pour enseigner son Moji no oshie est en ce sens extrêmement significatif des ruptures que celle-ci implique vis-à-vis des pratiques qui avaient cours jusque-là.

Sur nombre de ces points, Fukuzawa fait œuvre de précurseur et Moji no oshie apparaît comme l'un des tout premiers manuels modernes de la période du Gakusei. Un manuel tellement moderne d'ailleurs, que nombre de ses innovations ne seront pas reprises immédiatement et que certaines devront attendre plusieurs décennies avant de s'imposer et d'être généralisées (sans référence aucune à Fukuzawa et sous l'influence des pratiques occidentales essentiellement). Enfin, et surtout, la réflexion entreprise par Fukuzawa sur la lecture et sur son enseignement est inséparable des débats autour d'une langue écrite encore instable et des problèmes liés à l'apprentissage des kanji dans le Japon des années 1865-1875: la réforme de la langue écrite fut essentiellement pensée en terme d'éducation. Ce que montrent également très clairement les manuels sur lesquels nous allons maintenant nous arrêter.

Des manuels d'un nouveau type : une pédagogie pour entrer dans l'écrit

Jusqu'à la proclamation du Gakusei (1872), et pendant quelques années encore après celle-ci, certains enseignants utilisèrent comme supports pédagogiques, en attendant que soient publiés les premiers " vrais » manuels de lecture, des ouvrages d'un style nouveau, rédigés dans une langue plus simple et moins obscure que l'ancienne langue des lettrés - mais cependant difficiles d'accès car rédigés à l'attention d'un public adulte. Il s'agissait d'ouvrages contemporains, récits ou essais, ayant trait aux " choses de l'Occident ", tels que "L'habillement, la nourriture et le logement en Occident ", Seiyô ishokujû 西洋衣食住 (1868), de Katayama Junnosuke 片山淳之介 (ou Junkichi 淳吉), ou 
Gakumon no susume (1872) de Fukuzawa Yukichi, qui furent parmi les plus fréquemment utilisés. Nous ne nous attarderons pas ici sur ces ouvrages dont la description dépasse le cadre de cet article. Ils ne nous intéressent qu'en fonction de ce que représente leur adoption en tant que manuels, à savoir la volonté de rompre avec l'ensemble des anciens manuels et le manque de manuels d'un nouveau type dans les premières années de Meiji, ainsi que pour la façon dont ils étaient éventuellement utilisés - c'est-à-dire en tant que support à une pratique de la lecture de type sodoku.

Trois ouvrages, destinés aux enfants et d'une conception radicalement nouvelle, furent en revanche publiés entre 1868 et 1871 , trois parmi d'autres qui allaient dans la même direction mais avec moins de force et moins de cohérence. S'il est aujourd'hui très difficile de dire comment ils furent utilisés, sur quelle période et par quelle proportion d'enseignants, ils font néanmoins date dans l'histoire des manuels japonais en se démarquant définitivement des ouvrages utilisés jusqu'alors dans les écoles et en annonçant les futurs manuels pour débutants publiés après 1872 .

Il s'agit du Uhimanabi うひまなび,(Premières études), de Yanagawa Harukage 柳河春蔭 (1832-1870), publié en septembre 1868， du E.iri chie no wa 繪入知慧の環，(Les Anneaux magiques ${ }^{29}$ - ouvrage illustré) de Furukawa Masao 古川正雄 (1837-1877), publié en 1870 et du Chie no itoguchi ちえのいとぐち, (Amorce du savoir), du même auteur, publié l'année suivante. Conseillés en tant que manuels d'écriture par les directives officielles de 1872-73, ils furent très probablement les premiers manuels de l'époque contemporaine à contenir des matériaux pédagogiques rédigés à l'attention exclusive des débutants, les premiers également à tenter de présenter de façon « ordonnée » le système d'écriture de la langue japonaise.

\section{Le Uhimanabi (1868)}

Les dates de rédaction et de publication du Uhimanabi sont incertaines. Comme Yanagawa meurt en 1870 et que le département

${ }^{29}$ Ce terme désigne une sorte de "casse-tête chinois ", il est utilisé ici pour désigner un outil à même de développer l'intelligence des enfants. 
de l'Education cite son ouvrage dans ses directives de 1872, les spécialistes japonais considèrent qu'il s'agit très probablement de l'une de ses toutes dernières publications datant des années 1868-1870. Dans les directives officielles de $1872^{30}$, il apparaîtra en tant que manuel d'écriture (緅字 tsuzuriji lu kanazukai), au même titre que le Chie no wa ou le Chie no itoguchi de Furukawa. Il est donc tout à fait représentatif des manuels d'écriture que l'on destinait désormais aux élèves entrant pour la première fois à l'école.

Ecrit uniquement en kana, ce manuel de 28 pages se composait tout d'abord d'une présentation en tableaux de l'iroha (en hiragana puis en katakana), des chiffres chinois (de 0 à 10 puis cent, mille, dix mille et cent millions), des 50 sons (gojûin 五十音, présentés parallèlement en hiragana et en katakana) et des sons voisés, que suivaient des listes de mots composés de deux puis de trois syllabes (donc de deux puis de trois kana). Conçu sur le modèle des manuels de langue étrangère de l'époque, notamment d'anglais, il insistait sur la façon "d'épeler les mots". Les termes tsuzuriji ou kanazukai, littéralement : notation à l'aide de signes ou transcription en kana, furent d'ailleurs retenus en référence au mot anglais spelling (voir illustration $\mathrm{n}^{\circ} 3$ ).

Chaque katakana de la première colonne du tableau des cinquante sons - c'est-à-dire la colonne des cinq voyelles - était systématiquement combiné à tous les autres signes de ce même tableau pour former des mots de deux syllabes transcrits en katakana: aka, aki, aku, ake, ako, asa, etc. Les hiragana étaient ensuite présentés selon un procédé identique, mais moins systématique - seules les paires significatives étaient retenues - et dans l'ordre de l'iroha : iro, iha, iho, ihe, ito, ichi, etc., c'est-à-dire sans se limiter au cinq voyelles. Suivaient alors : roku, rofu, ron, haha, hato... Après avoir présenté de même les dakuon (濁音 sons voisés : $g a, g i, g u$, etc.) et les handakuon (半濁音 labiale : $p a, p i, p u$, etc.), le manuel reprenait la même présentation mais cette fois avec des mots de trois syllabes.

${ }^{30}$ Gakusei et Shôgaku kyôsoku 小学教則 (Directives d'enseignement pour les écoles élémentaires) de 1872. 


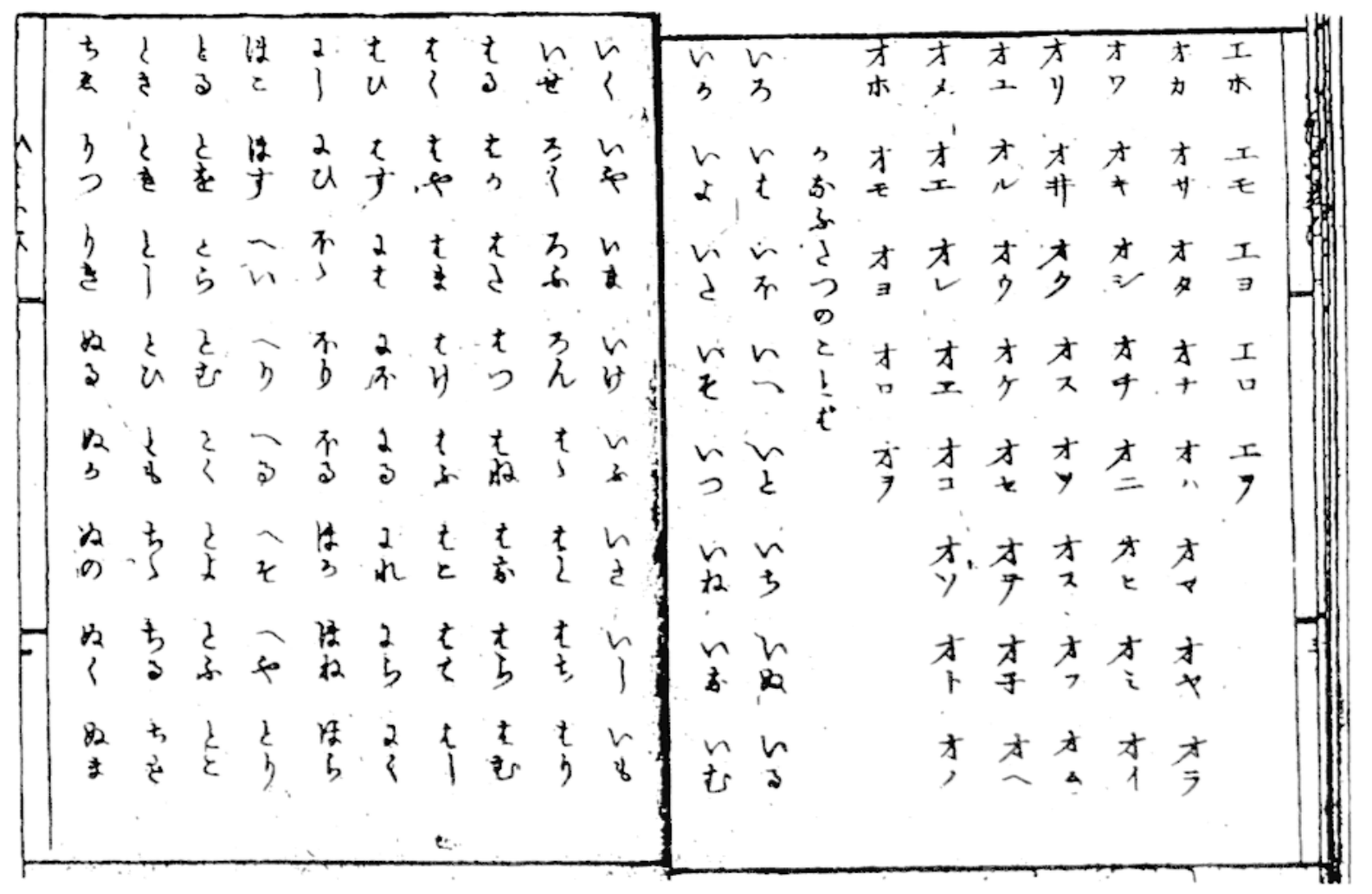

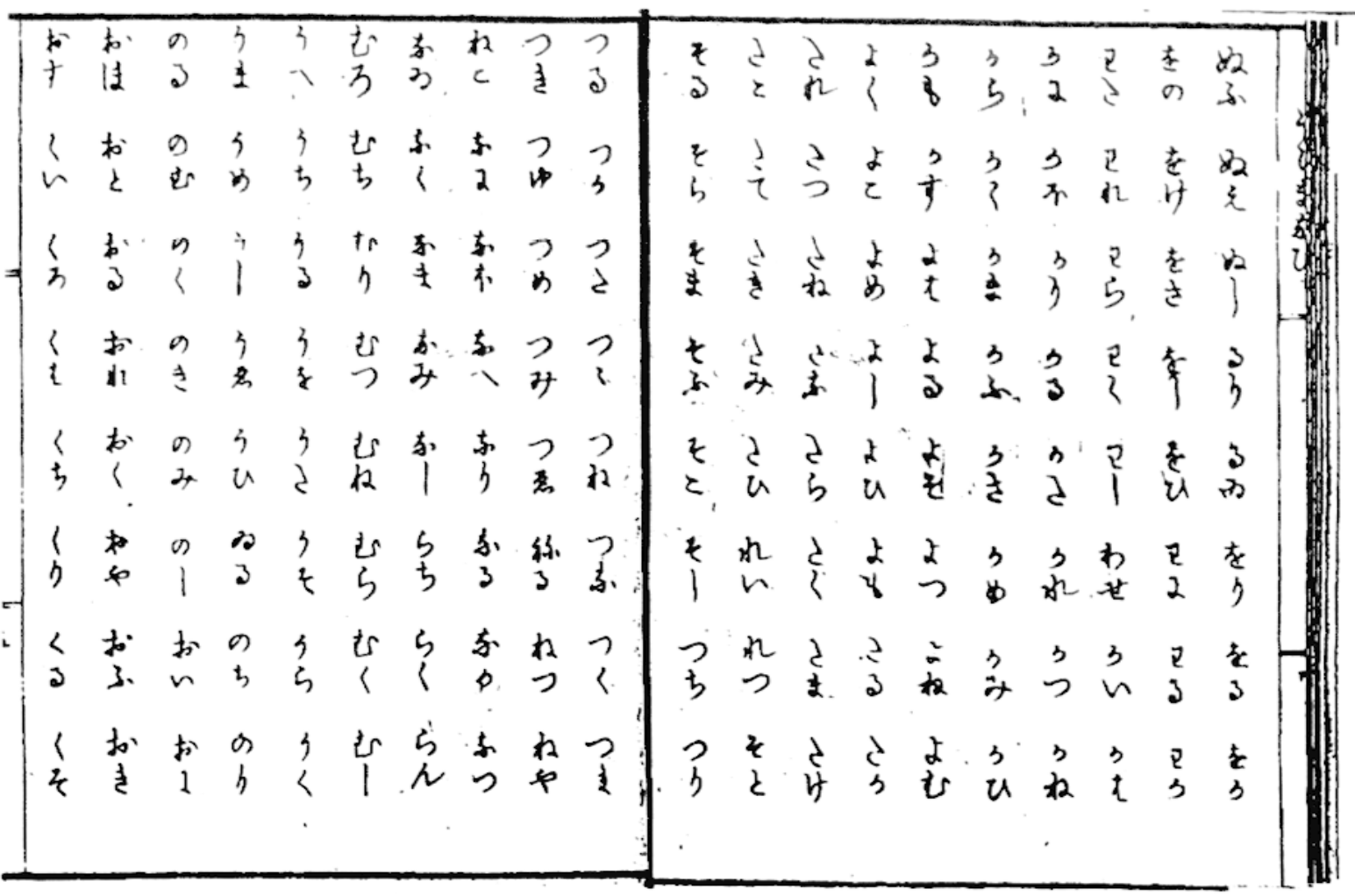

Illustration $\mathrm{n}^{\circ} 3-$ Uhimanabi (1868), p. 6-9. 
Ce manuel est un des tout premiers exemples d'une combinatoire "à la japonaise " enseignée de façon formelle et systématique. Entièrement consacré aux kana, le manuel faisait de leur acquisition le point de départ du processus d'enseignement de la lecture. Son contenu ne jouait pas du tout sur le registre du sens et «l'objet manuel » proposé aux élèves présentait beaucoup de ressemblance avec certains abécédaires occidentaux de l'époque que, par les différentes fonctions qu'il assuma ${ }^{31}$, Yanagawa dut certainement connaître. Le souci de systématisation est très net et fait date dans l'histoire de l'enseignement de la langue au Japon : signes isolés, mots de deux syllabes, mots de trois syllabes, sons simples, sons complexes, etc. Rares seront par la suite les manuels aussi « extrémistes » et aussi austères (aucune illustration) que celui-là.

\section{Le Chie no wa (1870) et le Chie no itoguchi (1871)}

Le Chie no wa fut rédigé par Furukawa Masao en tant que manuel pour l'apprentissage de l'écriture ${ }^{32}$. Il sera lui aussi mentionné dans les futures directives d'enseignement pour l'école élémentaire en tant que manuel pour l'enseignement de kanazukai à utiliser durant le premier trimestre de la première année de l'école élémentaire inférieure. C'est un manuel d'introduction à la langue écrite composé de mots ou d'expressions transcrits à l'aide de kana et de kanji "faciles " et illustrés de dessins. Quelques rudiments de grammaire sont également présentés. Le manuel est constitué de huit fascicules regroupés deux à deux en quatre tomes se présentant comme suit :

${ }^{31}$ Yanagawa fut notamment membre du Kaisei jo 開成所, institution créée par le bakufu pour centraliser et développer les "études occidentales", que le nouveau pouvoir rebaptisa ensuite Kaisei gakkô 開成学校. C'est dans ce cadre qu'il fut amené à participer à la réflexion sur le système scolaire.

${ }^{32}$ Les deux volumes du premier tome reçoivent l'autorisation d'impression en septembre 1870 et sont publiés le même mois. L'ensemble, gravé sur bois, est relié à la japonaise, la calligraphie est signée Uchida Fûzan* 内田楓山 et les dessins Hachida Shôn* 八田小雲 $\left[{ }^{*}=\right.$ lectures non attestées]. 
L'ÉBAUCHE D'UN NOUVEL ENSEIGNEMENT DE LA LANGUE ÉCRITE
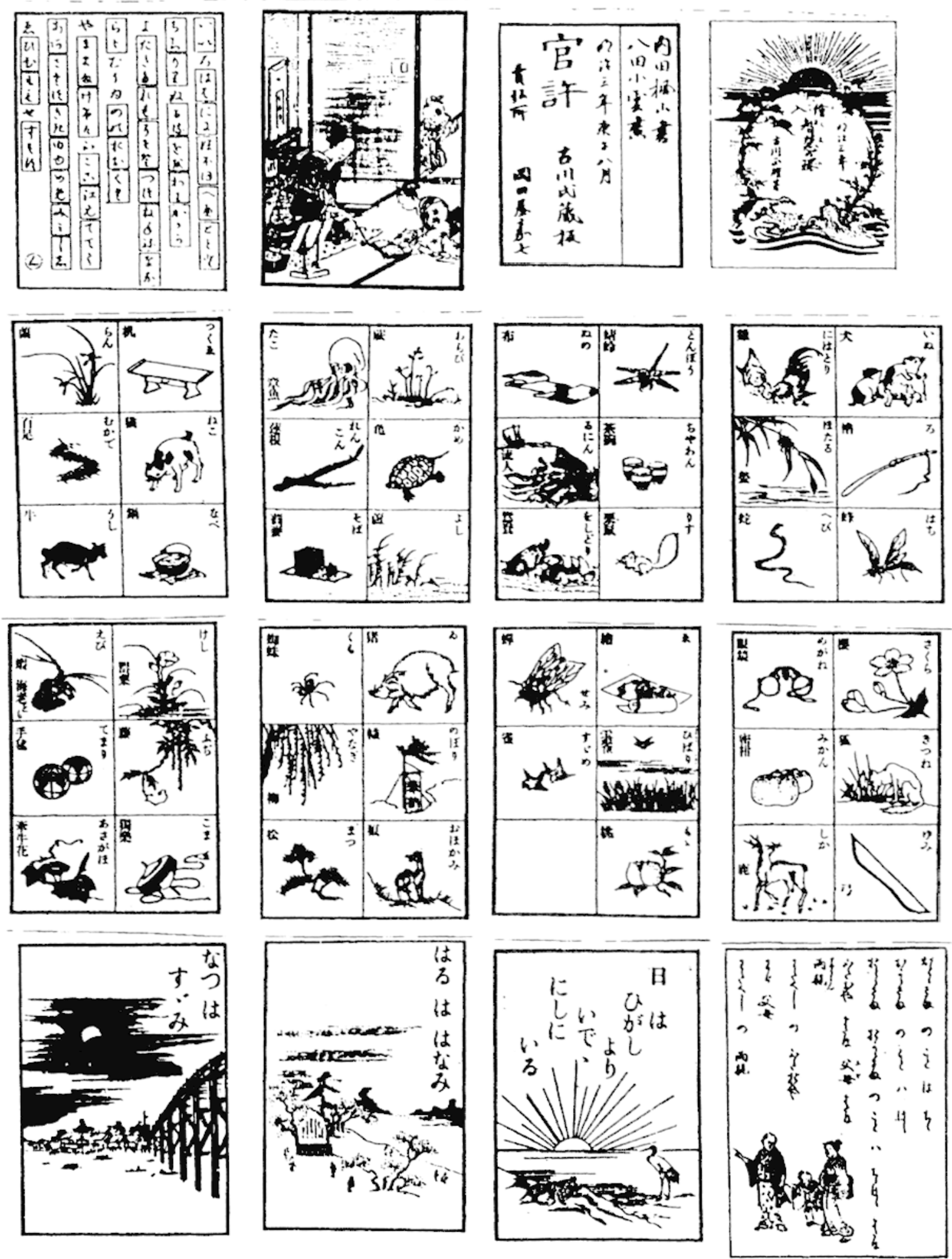

Illustration $n^{\circ} 4$-Quelques pages du Chie no wa (1870). 
TOME 1, FASCICule 1 (publié en août 1870) :

Iroha en hiragana ; iroha des sons voisés (nigori 濁) ; 47 vignettes contenant chacune un mot écrit en kanji, sa transcription en hiragana et un dessin $l^{\prime}$ illustrant (voir illustration $n^{\circ} 4$ ). Les mots - noms de choses ou d'animaux pris dans l'environnement quotidien des enfants - sont classés en fonction de leur première syllabe, dans l'ordre de l'iroha : inu, ro, hachi, niwatori, etc. Suivent les nombres, puis des expressions ou de courtes phrases dont la transcription en style cursif et en hentai gana $^{33}$ est assez difficile à lire. Les thèmes en sont : les parents, les points cardinaux et les directions, les éléments naturels, les dix « tiges célestes " et les douze signes horaires ou «branches terrestres 》 du calendrier chinois, ainsi que les mois de l'année, le tout illustré assez joliment par des dessins. A chaque fois qu'un kanji nouveau apparaît, le plus souvent seul et en tête des courtes phrases servant d'exemple, il est accompagné de furigana ${ }^{34}$ indiquant ses différentes lectures. Bizarrement, les kanji ainsi présentés ne réapparaissent pas forcément dans les phrases qui suivent et les mots dans lesquels ils auraient dû apparaître sont transcrits en hiragana. Le volume se termine sur des dessins, un ou deux par page, qui illustrent les différentes étapes de la culture du riz et décrivent les travaux dans les rizières. Chaque dessin est accompagné d'un très bref commentaire de quelques mots, parfois un seul, écrits en hiragana.

TOME 1, FAscicule 2 (publié en septembre 1870) :

Iroha et tableau des cinquante sons en katakana. Sons voisés, labiales. Tableau des 50 sons en hiragana. Suivent deux pages d'illustrations représentant des animaux, des objets familiers, des paysages, avec pour chaque mot le kanji utilisé habituellement et sa lecture en furigana : abaque, fil, pinceau, renard, oiseau, mer, rivière, etc. Le texte suivant encadre ces illustrations : "Aucun être humain

${ }^{33}$ Chaque kana, en fonction de son évolution propre depuis la création de ce système de transcription à partir du VIIf siècle, possédait plusieurs graphies appelées hentai gana 変体仮名.

${ }^{34}$ 振り仮名, kana de taille réduite pouvant apparaître au-dessus (dans l'écriture horizontale) ou à droite (dans l'écriture verticale) des kanji afin d'en préciser la lecture. 
n'ignore les noms des choses ordinaires telles que [celles qui sont représentées ici]. En revanche, très rares sont ceux qui savent que les mots ont aussi des "noms". Aussi vais-je maintenant les présenter ". Furukawa énumère donc les différentes catégories de mots : mots nominaux, pronoms personnels, mots de qualité, mots verbaux, mots adverbiaux, particules, conjonctions de coordination et mots exclamatifs. La présentation est chaque fois la même : numéro de la leçon, nom de la catégorie de mots que l'on va étudier, exemples illustrés de mots de cette catégorie (dessin + kanji + furigana), courte explication grammaticale tentant de définir la catégorie des mots en question. Après ces huit leçons d'initiation à la "grammaire ", ou plutôt à la terminologie grammaticale et aux différentes catégories de mots, le fascicule se termine par un " recueil de noms de marchandises importées ", présentant sur 16 pages, des objets, machines, vêtements, animaux ou autres " choses occidentales». Chaque objet dessiné est accompagné de son nom transcrit en hiragana et en kanji, ainsi que, pour les moins communs dans le Japon de Meiji, d'une très courte explication.

TOMes 2 A 4 (publiés entre septembre 1870 et 1872) :

Ces trois tomes rassemblaient des matériaux pédagogiques de toutes sortes destinés à des débutants. Le second fascicule de chacun d'entre eux était exclusivement consacré à la grammaire. Chaque tome était rédigé autour d'un thème général dans une présentation et une thématique qui empruntaient beaucoup aux ôraimono ${ }^{35}$. Le tome 2, “Tous les pays du monde "(Bankoku zukushi 万国尽), publié en novembre 1870, présentait dans son premier fascicule les noms et les drapeaux de divers pays, les " cinq races", les cinq continents, les cinq océans, etc., tandis que son second fascicule était consacré à la grammaire et à la piété filiale. Le tome 3 publié en 1872 et dans la préface duquel il était écrit : " il n'y a pas de honte à ne pas savoir écrire en enchaînant des phrases en chinois, mais il y en a à ne pas

${ }^{35}$ 往来物. On rassemble sous ce terme générique l'ensemble des ouvrages autres que les Classiques chinois qui furent utilisés durant les époques précédant l'ère Meiji comme manuels scolaires pour l'enseignement élémentaire. Cf. C. Galan (1998-a), op. cit., p. 33-39. 
savoir écrire des phrases en japonais " ${ }^{36}$, avait pour thème "L'inventaire des régions du grand Japon "(Dai nihon kuni zukushi 大日本国尽). “Les lieux célèbres" (meisho 名所) était celui du tome 4 publié en mai 1872 : après une brève introduction à l'histoire du Japon, étaient présentés les lieux historiques les plus célèbres du pays.

$\mathrm{Ce}$ manuel est un très bon exemple des tendances et des "tiraillements" de l'époque, qu'il s'agisse du contenu des enseignements ou du discours que l'auteur a jugé nécessaire $d^{\prime}$ introduire dans le corps même du texte. Rupture avec le kanbun ${ }^{37}$ et les classiques chinois pour se (re)centrer sur le Japon, ouverture sur la modernité, sur les mœurs et les techniques du monde occidental, mais continuité quant à la volonté de développer le sentiment de piété filiale et quant à la présentation qui rappelle celle de certains ôraimono. Dans le même temps pourtant, la simplicité et la clarté de la langue utilisée, proche du japonais parlé quotidiennement, font de ce manuel un support pédagogique radicalement différent de ceux-ci - on reconnaît là l'influence de son ami Fukuzawa et de leur maître à tous les deux, Ogata Kôan 緒方洪庵 (1810-1863).

$S^{\prime} y$ dessinent par ailleurs de nouvelles perspectives pour l'enseignement de la langue en général, celui de la lecture et de l'écriture en particulier. La plus intéressante est certainement le souci évident - beaucoup plus que dans le manuel de Fukuzawa par exemple - de créer un outil à l'attention des débutants et de l'adapter à leurs capacités, réelles ou supposées : progression du facile au difficile, en l'occurrence des hiragana aux kanji - sur ce point également la rupture avec les ôraimono ou les classiques est nette - ; présence permanente de furigana; volonté de prendre des " choses " proches du quotidien des enfants (sauf pour tout ce qui touche à l'Occident,

${ }^{36}$ On peut voir là un souci "nationaliste ", comme cela est écrit dans l'ouvrage dirigé par Karasawa Tomitarô 唐沢富太郎, Kyôiku jinbutsu jiten 教育 人物事典 (Dictionnaire encyclopédique des grands maîtres de l'éducation), 3 volumes, Tôkyô, Gyôsei ぎょうせい, 1984, vol. 1, p. 666, mais tout aussi vraisemblablement une nouvelle manifestation de la volonté de rompre avec les études chinoises.

${ }^{37}$ Les textes écrits en kanbun, c'est-à-dire en chinois, ont constitué jusqu'à l'époque moderne la référence fondamentale de l'écrit et du savoir. 
mais là le projet n'est plus pédagogique, il devient idéologique) ; introduction d'un grand nombre d'illustrations dans le but de faciliter l'étude par un contact plus direct de l'enfant avec la chose nommée, etc. L'aspect le plus étonnant du Chie no wa enfin consiste dans l'introduction de la grammaire, matière alors inconnue au Japon, à part égale avec l'écriture-lecture ${ }^{38}$.

Quant à la rédaction et à la présentation de l'ouvrage lui-même, si l'influence des manuels de langue pour débutants importés d'Occident est certaine, on peut aussi y déceler une continuité avec la façon dont étaient rédigés les manuels durant l'époque d'Edo, notamment dans la façon d'énumérer les mots et de les présenter classés par catégories sémantiques ou grammaticales.

Furukawa publia dans la même veine, l'année suivante, le Chie no itoguchi ちえのいとぐち, "Amorce du savoir», qui apparut aussi dans les directives officielles ultérieures en tant que manuel utilisable pour l'écriture (kanazukai) au cours du premier semestre de la première année de l'école élémentaire inférieure. Cet ouvrage présentait des mots en hiragana dans l'ordre de l'iroha, introduisait ensuite progressivement de courtes expressions puis des phrases très simples. Il fut rédigé en tant que manuel préalable au Chie no wa. Le fait que sa rédaction ait été ultérieure à celui-ci laisse supposer que, dans l'esprit de son auteur, il manquait une étape avant le Chie no wa et que la nécessité s'était fait sentir de proposer un manuel encore plus adapté aux débutants. Ce souci, qui l'amena à revenir sur la présentation habituelle des kana en proposant une progression : mots > expressions $>$ phrases, entièrement en kana, constitue une évolution capitale dans l'histoire des manuels de langue japonais ${ }^{39}$.

${ }^{38}$ Sur ce point également, l'influence de Fukuzawa dut être déterminante, si l'on se réfère à ce que celui-ci écrivait de la matière tokuhon dans son livre Keimô tenarai no fumi (cf. supra), que Furukawa ne pouvait que connaître.

${ }^{39}$ Le Chie no itoguchi utilisait une langue très ordinaire. A côté des formes classiques ari ou nari, apparaissaient dans certaines leçons les suffixes verbaux -masu, -mashita et -mashô qui relevaient de la langue «vulgaire ». L'influence de Fukuzawa et surtout d'Ogata Kôan qui prônait à ses élèves la simplicité et la compréhensibilité apparaît une fois encore déterminante (voir N. Twine, op. cit., p. 86). 
L'étude de l'enseignement de la lecture au cours de l'époque $\mathrm{d}^{\prime} \mathrm{Edo}^{40}$ et des toutes premières années de l'ère Meiji permet de bien cerner les deux courants de force inégale qui, à la veille de la proclamation du Gakusei, traversent le monde de l'enseignement de la langue et plus généralement celui de l'éducation.

Le premier, souterrain, profond, est issu de l'évolution proprement japonaise, sur une dizaine de siècles, $d$ 'un ensemble de valeurs et de pratiques héritées de l'enseignement classique de la Chine et de la tradition confucéenne. L'enseignement de la lecture et de l'écriture qui relève de ce courant n'a guère évolué depuis l'époque de Heian qu'en fonction des changements qui ont affecté le japonais écrit. Ses principes fondamentaux n'ont jamais été remis en cause : au tout début de l'ère Meiji, la méthode " classique " est toujours la seule à être appliquée sur le terrain.

Le second courant, minoritaire mais «brillant », puise sa vitalité et sa légitimité dans les débats sur la modernisation du pays qui agitent alors la société japonaise. Il s'est constitué sur la critique, radicale, du modèle classique de l'éducation dont il conteste les finalités et les méthodes - inadaptées à la société moderne. Ce courant de pensée, qui ouvre de nouvelles pistes pour la réflexion pédagogique, donnera au Japon son premier système éducatif moderne. Les valeurs et les méthodes « classiques " n'en disparaîtront pas pour autant et le modèle " classique " de la transmission du savoir perdurera ainsi bien après l'instauration du nouveau système. Car si, sur le plan du discours et des débats, le courant « rénovateur » est en pleine effervescence, sur le terrain, en revanche, il reste inexistant et ou plutôt tarde à se réaliser dans sa dimension pédagogique " pratique ".

L'historien de l'éducation Hida Takio (1907-1991) regroupe sous la dénomination générale de kobun kyôju 古文教授, littéralement " l'enseignement des classiques (ou des textes anciens) ", l'ensemble des pratiques héritées des terakoya et des écoles des fiefs, hankô 藩校, dont il confirme le caractère dominant au début de Meiji.

${ }^{40}$ Cf. C. Galan (1998-b), op. cit. 
La caractéristique fondamentale de ces pratiques réside dans le principe de la « lecture-récitation 》, sorayomi anshô shugi 空読暗誦主義, sur lequel elles reposent. La lecture de type sodoku, qui permet d'accéder " magiquement " au sens par la répétition, en est la forme scolaire la plus représentative et la plus essentielle.

Hida caractérise la méthode " classique " de la façon suivante ${ }^{41}$ : 1 - C'est une méthode qui se centre sur la pratique de la lecture à haute voix (dokushô 読誦) et qui ne tient quasiment aucun compte du sens ou de l'esprit du texte.

2 - La compréhension ne peut résulter que d'un commentaire superficiel des mots ou des expressions, pris un à un.

3 - Une très grande importance est accordée au " par cœur » et à la récitation dans les exercices de répétition.

Soulignons encore plusieurs points. C'est une méthode qui ne prend pas en compte les besoins et les intérêts de l'enfant, de l'apprenant, qu'elle considère non pas en tant que tel, mais en tant que futur adulte. La réflexion sur l'ordre, la forme et la cohérence des leçons par rapport à leur objet et à leur(s) objectif(s) se limite à une présentation des supports de lecture qui s'efforce d'aller des plus abordables aux plus obscurs. La plus grande partie des supports de lecture utilisés sont d'une très grande complexité, à la fois hermétiques et archaïques : très peu d'entre eux furent rédigés à l'attention particulière des débutants (aucune prise en compte du niveau de difficulté des matériaux pédagogiques utilisés et de leur degré $d^{\prime}$ adaptation aux enfants). Les leçons sont centrées sur l'enseignant qui décide seul du rythme de la progression et des matériaux utilisés : leur forme est, au sein de chaque école, figée et sans souplesse, quasiment immuable, sinon " rituelle ». Hida Takio décrit de la façon suivante une leçon de lecture type dans les premières années de Meiji ${ }^{\mathbf{4 2}}$ :

L'enseignant indiquait d'abord l'objectif de ce qu'il allait enseigner durant la leçon. Il lisait ensuite lui-même d'un bout à l'autre

${ }^{41}$ Hida Takio 秋田多亳雄，Kokugo kyôiku hôhô ron shi 国語教育方法諭史 (Histoire des méthodes d'enseignement de la langue japonaise), Tôkyô, Meiji tosho 明治図音, 1966, p. 26.

${ }^{42}$ Ibid. 
[tsûdoku 通読] l'ensemble du texte (parfois en le découpant). Les élèves écoutaient attentivement. Quand la lecture du maître était terminée, chaque élève lisait le texte d'un bout à l'autre [tsûdoku] ou encore des élèves lisaient le texte à tour de rôle [rindoku 输読]. Quand l'exercice était terminé, on passait au cours/interprétation du professeur [kôgi 㸷義]. Quand le cours était terminé, les élèves commençaient l'exercice appelé rinkô [输陆]. C'est-à-dire que les meilleurs, ou les plus anciens, d'entre eux refaisaient à l'attention des plus jeunes ou de ceux qui étaient en retard le commentaire du professeur en l'imitant jusque dans le ton. Celui-ci pouvait également, dans certains cas, faire faire une dictée avant ou après la lecture en sodoku.

Cette description ne doit pas faire oublier que sur le plan de la pratique pédagogique proprement dite, la primauté, dans le modèle classique jusqu'à la Restauration de Meiji, était accordée à l'écriture dont l'apprentissage constituait de fait la première étape de celui de la lecture. L'enseignement de l'écriture débutait par les syllabaires et les nombres. Les kanji étaient ensuite étudiés au travers de la copie répétitive de modèles d'expressions ou de phrases. Les signes, kana ou kanji, étaient vus le plus souvent un par un, hors de tout contexte véritablement significatif. Dans la période des apprentissages premiers, comptait avant tout la "mémoire de la main ", jugée indispensable à leur assimilation correcte et définitive. Pendant toute la période " élémentaire " de la formation, c'est-à-dire, en gros, tout le temps où la pratique de la lecture de type sodoku occupait la plus grande partie de l'étude (jusque vers 12 ou 14 ans), la compréhension était considérée au mieux comme seconde, sinon comme inutile. Seul un déchiffrage à haute voix correct était visé.

L'activité d'écoute jouait un rôle important dans l'apprentissage de la lecture. Les élèves débutants étaient invités à écouter les textes lus par leurs aînés pour s'en imprégner et commencer à les mémoriser. On considérait comme parfaitement inutile de savoir si l'enfant comprenait ou pas ce qu'il lisait étant donné qu'il était justement en train d'apprendre à lire, et ce principe avait pour corollaire qu'il était donc tout aussi inutile de lui proposer des textes qui pourraient l'intéresser, puisque de toute façon il ne les comprendrait pas. 
La " compréhension ", quand elle était recherchée - ce qui était loin d'être toujours le cas -, découlait de l'explication du maître ou de l'un des étudiants plus âgés : elle était transmise de ceux qui savent à ceux qui ne savent pas et c'était là la voie normale de l' " acquisition ", ou plutôt de la transmission, du savoir. L'autre voie d'accès au sens $d^{\prime}$ un écrit était la lecture/récitation répétée de celui-ci des dizaines et des dizaines de fois. Dans ce dernier cas, la compréhension s'acquérait seule et était le fruit d'une activité mécanique. Les maîtres-mots de l'enseignement de la lecture étaient donc répétition et mémorisation, et c'est cette dernière qui était évaluée à l'examen. Récitation et lecture à haute voix, individuelles ou en groupes, étaient, en classe, les activités de lecture par excellence.

La progression était programmée de façon assez stricte : tant de caractères, de versets ou de phrases par jour à tel âge ou, plus fréquemment encore, à telle étape de l'étude, indépendamment de l'âge. Les manuels, ou ce qui en tenait lieu, étaient à la fois support de lecture et de morale. Enfin, la notion de tradition était très forte dans cet enseignement de la langue : peu de maîtres ou d'érudits s'interrogeaient vraiment sur le pourquoi et le comment de la méthode utilisée.

Au total, c'est une méthode qui " marchait ", dans le sens où les élèves qui recevaient cet enseignement apprenaient effectivement à déchiffrer les textes qu'on leur proposait : certains d'entre eux - ceux de la classe des guerriers essentiellement - parvenaient même à accumuler les savoirs. Il est difficile cependant de connaître le niveau de lecture que les enfants des terakoya pouvaient acquérir en - au mieux - trois ou quatre années de scolarité irrégulière. Que signifiait « savoir lire » au cours de l'époque d'Edo ? La question n'a, semble-t-il, jamais été posée ${ }^{43}$.

Cette méthode en tout cas montra aussi ses limites, dès lors que refusant la logique élitiste on se soucia de l' "alphabétisation " des

${ }^{43} \mathrm{Cf}$. Richard Rubinger, " Problems in Research on Literacy in $19^{\text {th }}$ Century Japan ", Nihon kyôiku shi ronsô - Motoyama Yukihiko kyôju taikan kinen ronbun shî 日本教育史論丵 本山幸彦教授退官記念論文集 (Sur l'histoire de l'éducation au Japon - Mélanges offert à Motoyama Yukihiko à l'occasion de son départ à la retraite), Tôkyô, Shin bungaku shuppan 新文学出版, 1988, p. 568-545. 
masses : fondée sur le mode individuel, elle n'était pas applicable à une grande échelle. Par ailleurs, l'écart était beaucoup trop grand entre les efforts qu'elle réclamait, le temps qu'elle requérait, et les capacités et les préoccupations des enfants toujours plus nombreux auxquels elle s'adressait, ainsi que l'investissement que leurs parents voulaient ou pouvaient consentir. A la veille de Meiji, l'enseignement individualisé n'était plus à même de corriger les travers (routine, ennui, abrutissement) de la méthode classique, en permettant à l'enseignant d'adapter son cours aux capacités et au niveau de chaque enfant.

Avec la diffusion de la lecture dans la population au cours de la seconde moitié de l'époque d'Edo, l'idée que tout le monde peut apprendre à lire à condition de suivre la progression normale et de travailler sans relâche se répand. La difficulté rencontrée par les maîtres pour apprendre à lire à un nombre croissant d'individus amène alors certains esprits éclairés à réfléchir non seulement sur les méthodes d'enseignement et leur amélioration possible mais également sur la langue écrite. C'est ainsi, qu'au nom de l'accès de tous à l'écrit, certains occidentalistes - appelons-les ainsi pour simplifier - remettent en cause et la méthode et la finalité (l'étude, c'est la lecture) de l'enseignement "classique " de la lecture, et aussi ce qui les conditionnait toutes les deux : la langue écrite elle-même, qui, coupable à leurs yeux d'une trop grande complexité, doit être simplifiée. Dans un premier temps toutefois leurs idées ne dépassent pas les cercles intellectuels. Les instituteurs après la Restauration sont les mêmes que ceux de l'époque d'Edo, maîtres des terakoya ou des hankô, formés aux anciennes pratiques. Ils continuent d'enseigner comme ils l'ont toujours fait avec les anciens "manuels" tout d'abord, avec les nouveaux ensuite - plus ou moins bien adaptés, plus ou moins bien compris - pour ceux qui peuvent se les procurer.

L'enseignement de la lecture dans les toutes dernières années de l'époque $d^{\prime} E d o$ et les toutes premières de l'ère Meiji, jusque vers 1871, est au dernier stade de son évolution " purement" japonaise - c'est-à-dire hors de toute influence étrangère (voir schéma $\left.n^{\circ} 1\right)$ - ou presque. La méthode sur laquelle il repose et que nous avons appelée ici " méthode classique " possède, à la veille de Meiji, 
la plupart des caractéristiques des méthodes « traditionnelles " ayant cours en Occident à la même époque.

Comme ces dernières ${ }^{44}$, elle repose sur des manuels dont l'enseignement s'effectue page à page. Elle est adulto-centrée, l'enseignant décide de tout : du rythme, des supports de lecture, de la valeur des performances, etc. Elle repose sur une acquisition mécanique de la lecture qui favorise la lecture à haute voix et se fonde sur la répétition et la mémorisation. Elle se distingue toutefois de ces méthodes traditionnelles sur deux points importants : (1) l'écriture est enseignée avant la lecture (au contraire de la tradition occidentale qui veut que l'on n'apprenne à écrire que lorsqu'on sait lire), et (2) elle ne progresse pas véritablement de manière " synthétique " de l'élément au tout, du signe à la phrase, c'est-à-dire par synthèse d'éléments nouveaux avec d'autres déjà étudiés, d'une façon rigoureusement systématique du simple au complexe. La progression signes $>$ mots $>$ phrases $>$ textes n'est guère perceptible que dans les tout premiers temps de l'apprentissage de l'écrif ${ }^{45}$. Et il faudrait nuancer encore en ajoutant que les kana et les kanji ne sont pas véritablement présentés des plus simples (ou des plus fréquents) aux plus complexes, mais le sont soit dans l'ordre de l'iroha, soit en fonction de leur " rencontre » dans des textes lus, soit encore en fonction de critères

${ }^{44}$ Voir Guy Palmade, Les Méthodes en pédagogie, Paris, PUF, $15^{e}$ édition, 1994, p. 9-11.

${ }^{45}$ A la même époque, en France et dans les pays européens : « [Tous les plans d'études et les emplois du temps de l'école primaire], ou presque, se basent sur une méthode synthétique qui part des éléments du mot pour atteindre le mot lui-même, puis la phrase, avant de parvenir à la lecture courante. Un bon exemple de cette méthode nous est fourni par A. Rendu, en 1819, qui prévoit neuf sortes de "leçons" pour l'apprentissage de la lecture. "La table d'alphabet, la table des syllabes, le syllabaire, le second livre pour apprendre à épeler et à lire par syllabes, le même second livre dans lequel ceux qui savent épeler commencent à lire, le troisième livre qui sert à apprendre à lire par pauses, le psautier, la Civilité chrétienne, les lettres écrites à la main." " ; Pierre Giolitto, Histoire de l'enseignement primaire au XX $\mathrm{X}^{e}$ siècle, 2 tomes, Paris, Nathan, 1984, tome 2, p. 13. 


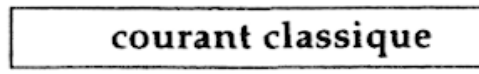

DOMINANT

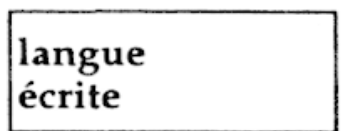

principes fondamentaux

"Si on lit cent fois, on

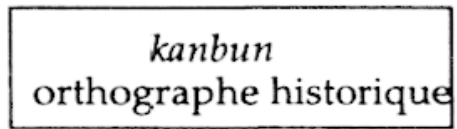
comprend" + étudier = lire lecture propédeutique de la vertu

\begin{tabular}{l|}
\hline manuels \\
\hline finalité \\
\hline $\begin{array}{l}\text { mode } \\
\text { d'enseignement }\end{array}$ \\
\hline $\begin{array}{l}\text { pratiques } \\
\text { pédagogiques }\end{array}$ \\
\hline progression \\
\hline méthode \\
\hline
\end{tabular}

mots-clés

particularités parvenir à bien déchiffrer

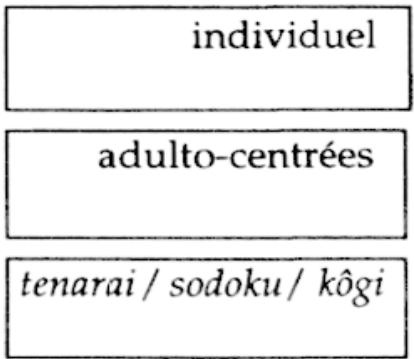

globale / cumulative

répétition et mémorisation lecture à haute voix

importance de l'écoute au début de l'apprentissage courant " rénovateur »

MINORITAIRE

simplification de l'écrit langue proche de la langue parlée

lire dans un but pratique : lecture-outil ; lecture préalable du savoir et de l'indépendance

nouveaux, surprenants une dizaine à peine

parvenir à comprendre

tend vers le simultané

adulto-centrées / intérêt naissant envers l'apprenant

ombinatoire des syllabaires / mots / phrases courtes/textes

tend vers le synthétisme du simple au complexe

répétition et mémorisation lecture à haute voix

\begin{tabular}{|c|}
\hline $\begin{array}{c}\text { légère influence occidentale } \\
\text { discours rénovateur mais } \\
\text { pratiques encore } \\
\text { fortement " classiques" }\end{array}$ \\
\hline
\end{tabular}

Schéma $n^{\circ} 1$ - Les données fondamentales de l'enseignement élémentaire de la lecture à la veille de la promulgation du Gakusei (1872). 


\section{L'EBAUCHE D'UN NOUVEL ENSEIGNEMENT DE LA LANGUE ECRITE}

tels que l'importance morale ou culturelle de certains caractères étudiés en prioritét

Le souci de progresser du simple au complexe ne concerne que les classiques chinois ou les supports de la lecture de type sodoku que l'on veille à aborder à partir des plus simples pour aller ensuite vers les plus obscurs. Corollaire de cette démarche, les élèves étaient mis très tôt en contact avec de véritables écrits d'adultes, même si ceux-ci leur étaient, dans un premier temps au moins, parfaitement incompréhensibles.

La méthode ne peut pour autant être définie comme " analytique" - l'autre alternative au sein des méthodes dites "traditionnelles "-car si elle part bien, avec le sodoku, de la " matière à lire dans son ensemble $"{ }^{47}$, elle ne débouche sur aucune décomposition ou analyse en mots puis en signes. Ni "synthétique ", ni " analytique ", les adjectifs " globale " et "cumulative " ${ }^{48}$ nous semblent la caractériser avec plus de justesse ${ }^{49}$. C'est pourquoi, afin d'éviter toute confusion avec la terminologie actuelle, nous avons choisi de nous en tenir à l'adjectif "classique", réservant «traditionnel » aux méthodes postérieures au Gakusei ${ }^{50}$.

Dans les dernières années de l'époque d'Edo et le tout début de l'ère Meiji, trois lignes de force, qui seront désormais des constantes du fait éducatif japonais, traversent ainsi la réflexion et les pratiques pédagogiques:

${ }^{46}$ Voir par exemple les caractères-mots proposés par Kaibara Ekiken, cf. C. Galan (1998-b), op. cit., p. 18-19.

${ }^{47}$ Th. Braun, Cours théorique et pratique de pédagogie et de méthodologie, 3 vol., Liège, 1872 (cité par P. Giolitto, op. cit., tome 2, p. 19).

${ }^{43} C^{\prime}$ est-à-dire procédant par assimilation "globale " et " cumulative " des caractères, des mots ou des séquences/phrases lus.

19 Il semble de toute façon très difficile de distinguer, sur toutes ces questions, ce qui relevait d'une volonté pédagogique clairement définie et ce qu'imposaient la nature particulière de l'écrit japonais et son évolution.

${ }^{50} \mathrm{Cf}$. Christian Galan, L'Enseignement de la lecture au niveau élémentaire dans le système éducatif du Japon moderne depuis Meiji (1872-1992), thèse de doctorat nouveau régime, études japonaises, sous la direction du professeur Jean-Jacques Origas, INALCO, Paris, juillet 1997. 
1 - Les principes et les pratiques « classiques ».

2 - La réflexion pédagogique contemporaine des enseignants japonais.

3 - Les idées et les méthodes occidentales (qui commencent à être introduites).

C'est de leur convergence et de leur confrontation que naîtra le nouveau système d'éducation japonais. Pendant un temps toutefois, ce système n'aura de nouveau que le nom et sa structure - sa " coquille " - institutionnelle. Sur le terrain, les hommes de l'époque précédente continuent d'enseigner avec les mêmes méthodes et les mêmes " manuels » jusque bien après la promulgation du Gakusei.

Au-delà des intentions et des discours des intellectuels ou des responsables de l'époque, les premières années de Meiji (1867-1885) offriront, sur le plan de la pratique pédagogique dans les écoles, plus de continuité avec la fin de l'époque d'Edo qu'avec la période qui s'ouvrira avec les années $1885-86$ et qui verra se mettre en place un véritable système éducatif national, centralisé et (de plus en plus) uniformisé. Et même alors, les pratiques pédagogiques « classiques " ne disparaîtront pas totalement pour autant : elles se mêleront en un syncrétisme original aux nouvelles conceptions et aux nouvelles méthodes développées à partir de ce qui se fait en Occident. Ce syncrétisme se réalisera certes le plus souvent au détriment de ces dernières, mais finira par constituer le fondement de ce que l'on appelle aujourd'hui avec dédain, crainte ou respect - mais sans toujours bien le connaître - « l'enseignement à la japonaise ». 


\title{
BIBLIOGRAPHIE COMPLEMENTAIRE
}

\begin{abstract}
AKITA Kisaburô 秋田喜三郎, Shotô kyôiku kokugo kyôkasho hattatsu shi 初等教育国語教科書発達史 (Histoire du développement des manuels de langue japonaise de l'éducation élémentaire), Hiroshima, Bunka hyôron 文化評論, 1977.
\end{abstract}

FUKUZAWA Yukichi 福沢諭吉, Fukuô jiden 福翁自伝 (La vie du vieux Fukuzawa par lui-même), Tôkyô, Iwanami bunko 岩波文庫, 1993 (1 (ère $^{\text {en }}$ éd. : 1899).

FURUTA Tôsaku 古田東朔, Shôgaku tokuhon benran 小學讀本便覧 (Guide des manuels de lecture de l'école élémentaire), 10 tomes +1 tome complémentaire, Tôkyô, Musashino shoin 武蔵野書院, 1978 [dans cette série, les manuels sont reproduits en fac-similé dans leur intégralité ; les manuels étudiés dans cet article figurent dans le tome complémentaire].

KAIGO Tokiomi 海後宗臣 (sous la dir. de), Nihon kyôkasho taikei kindai hen 日本教科書体系 近代編 (Panorama des manuels scolaires japonais - époque contemporaine), 27 tomes, Tôkyô, Kôdansha 講談 社, 1964-1967 [les manuels étudiés dans cet article figurent dans le tome 4].

Kokugo kyôiku kenkyû-jo 国語教育研究所 (Institut de recherche sur l'enseignement de la langue japonaise) (éd.), Kokugo kyôiku kenkŷ̂ dai jiten 国語教育研究大辞典 (Grand dictionnaire de la recherche sur l'enseignement de la langue japonaise), Tôkyô, Meiji tosho 明治図書, 1991.

KUWABARA Saburô 桑原三郎, Fukuzawa Yukichi 福沢諭吉 (Fukuzawa Yukichi), Tôkyô, Maruzen raiburarî 丸善ライブラリー, n 48, 1992. 
NISHIKAWA Shunsaku 西川俊作, Fukuzawa Yukichi no yokogao 福沢諭 吉の横顔 (Fukuzawa Yukichi de profil), Tôkyô, Keiô gijuku daigaku shuppan kai 慶応義熟出版会, 1998.

Tôkyô hôrei 東京法令 (éd.)，Kokugo kyôiku shi shiryô 国語教育史資料 (Documents pour l'histoire de l'enseignement de la langue japonaise), collectif, 6 volumes, Tôkyô, 1981 [vol. 2 : Manuels scolaires].

WATANABE Tokusaburô 渡辺徳三郎, Fukuzawa Yukichi-katei kyôiku no susume 福沢諭吉・家庭教育のすすめ (Fukuzawa Yukichi, promoteur de l'éducation familiale), Tôkyô, Shôgakukan 小学館, 1985.

YOSHIDA Yutaka 吉田豊, Edo kana komonjo nyûmon 江戸かな古文書入 門 (Introduction aux ouvrages en kana de l'époque d'Edo), Tôkyô, Kashiwa shobô柏書房, 1995. 